\title{
Scalar transport in random cylinder arrays at moderate Reynolds number
}

\author{
By BRIAN L. WHITE AND HEIDI M. NEPF \\ Department of Civil/Environmental Engineering, Massachusetts Institute of Technology, \\ Cambridge, MA 02139, USA \\ blw@mit.edu; hmnepf@mit.edu
}

(Received 19 August 2002 and in revised form 21 January 2003)

This paper theoretically describes and experimentally verifies two mechanisms leading to longitudinal dispersion of a passive tracer in a random array of circular cylinders. We focus on moderate Reynolds numbers of order 10-1000, specifically the range characterized by unsteady cylinder wakes. In this regime, two mechanisms contribute to dispersion, each associated with a distinct region of the cylinder wakes: (i) the unsteady recirculation zone close to each cylinder, and (ii) the velocity defect behind each cylinder, which extends downstream of the cylinder over a distance of the order of the cylinder spacing. The first mechanism, termed vortex-trapping dispersion, is due to the entrainment of tracer into the unsteady recirculation zone, where it is momentarily trapped and then released. A theoretical expression for this dispersive mechanism is derived in terms of the residence time and size of the recirculation zone. The second mechanism is due to advection through the random velocity field created by the random distribution of the wake velocity defect. We derive an expression for the defect behind an average cylinder, and show that it decays owing to array drag over a length scale called the attenuation length, which is of the order of the cylinder spacing. The superposition of the wake defect behind each cylinder creates the random velocity field. Theoretical predictions for dispersion agree very well with observations of tracer transport in a laboratory cylinder array, correctly capturing the dependence on array density and Reynolds number. The laboratory studies also document a transition in small-scale mixing at cylinder Reynolds number $\approx 200$. Below this limit, individual filaments of tracer remain distinct, producing significant fluctuations in the local concentration field. At higher Reynolds number, cylinder wakes contribute sufficient turbulence to erase the filament signature and smooth the tracer distribution.

\section{Introduction}

Arrays of solid bodies fixed relative to an oncoming flow are found in a number of physical situations including fixed bed reactors, porous media, aerosol filtration, building clusters in urban environments, and plant canopies. In each case, it is often important to predict the transport of a passive scalar species in the array. However, owing to the spatial and often temporal complexity of the velocity field, application of the scalar transport equation at the scale of individual array elements is prohibitive. It is thus desirable to derive parameters describing transport on macroscopic scales, i.e. scales greater than the detailed geometry of the array.

Here, we specifically consider the problem of longitudinal dispersion in moderateReynolds-number flow through a randomly distributed array of circular cylinders. 
This model provides a good description of aquatic canopies found in freshwater wetlands and saltwater marshes. Such canopies often consist of plants with cylindrical stem morphology, such as Spartina alterniflora (smooth cordgrass) or Juncus roemerianus (needle rush). For these aquatic systems, the Reynolds number based on stem diameter may range from $R e=10$ to 1000 , which includes the laminar flow regime for which plant wakes are steady as well as the regime of unsteady wakes with periodic vortex shedding. In this study, we restrict our attention to the unsteady range, typically above $R e \approx 40$. Moreover, we consider emergent canopies, with stems piercing the water surface, by adopting as our model a two-dimensional array of circular cylinders. We derive analytical descriptions for two mechanisms of longitudinal dispersion associated with two distinct regions of each cylinder wake: (i) the unsteady recirculation zone, and (ii) the velocity defect behind each cylinder extending downstream over a distance of the order of the cylinder spacing. A comparison to experimental observations confirms that these are the dominant mechanisms of dispersion in the array. To our knowledge, this is the first description of the longitudinal dispersion caused by unsteady flow in a moderate-Reynoldsnumber array.

Previous studies of dispersion in random arrays have focused primarily on vanishing Reynolds number for which the flow is steady and described well by Stokes theory. For Stokes flow through a random array of spheres, Koch \& Brady (1985) derived an analytical expression for the mechanical dispersion which results from advection of particles through the heterogeneous structure of the array. Here, 'mechanical' defines a dispersive process that is independent of diffusion, i.e. advection sets the dispersive time scale. Koch \& Brady also deduced a non-mechanical component of dispersion that arises from the trapping of scalar in the boundary layer near each particle surface, which they termed 'hold-up dispersion', and which we will refer to as 'boundary-layer dispersion'. The magnitude of this component depends on molecular diffusion, which controls the time scale for hold up.

Eames \& Bush (1999) predicted dispersion around bodies of arbitrary shape based on potential flow theory. By considering the distortion of a material surface around the body, they derived a mechanical dispersion coefficient proportional to the added mass, $C_{m}$, and a distortion length scale, $L$. This treatment predicts the dispersion induced by streamline curvature around cylinders. However, as it relies on inviscid theory, it does not address the significant effect of flow separation and vortex formation, which results in trapping of scalar in the periodic primary wake. Nor does it account for the suppression of velocity in the wake of the cylinder elements. Both of these effects are considered in this study.

Transverse diffusion at moderate to high Reynolds number has been studied by Nepf (1999) who derived an expression for transverse mechanical diffusion. In the same study, Nepf used scaling arguments to predict the turbulence production within a cylinder array, which in turn was used to predict turbulent diffusion. Together the predicted mechanical and turbulent diffusion accounted for the observed array-scale transverse diffusion measured in the range $R e=400 \sim 2000$.

However, no analysis has yet considered the effects of separation and unsteady wakes on longitudinal dispersion in moderate-Reynolds-number arrays. It will be shown here that both the unsteady recirculation zone and the velocity defect behind each cylinder contribute to dispersion. First, dispersion due to the recirculation region is derived in terms of the recirculation zone size and residence time. Then, an expression for the velocity defect behind an average cylinder is obtained by averaging the momentum equations, with a thin-wake approximation, and accounting for the 
effects of drag from the array. The defect is found to decay owing to drag over a scale that we call the attenuation length. Far from the cylinder, the momentum equation is approximately linear, and the superposition of all wake defects composes a random velocity field that creates tracer dispersion.

In $\S 2$, an array-scale description of dispersion is found from a spatial and temporal average of the scalar conservation equation. In $\S 3$, we derive a dispersion constant describing the contribution of entrainment and subsequent release of scalar in the recirculation zone. In $\S 4$, we derive an expression for the velocity defect in the wake of a single cylindrical element and use it to deduce the dispersive contribution of an array of distributed elements. In $\S \S 5$ and 6 , we report experimental observations of longitudinal dispersion of tracer in a random array of cylinders. A comparison of the experimental and theoretical results gives support to the theory and suggests that the combined effects of the unsteady recirculation region and the velocity defect in each wake make the dominant contribution to dispersion in the array.

\section{Model description}

The array we consider is two-dimensional, infinite in extent, and defined by the two-dimensional coordinate system $\boldsymbol{x}=x \boldsymbol{i}+y \boldsymbol{j}$. The array contains cylinders of constant diameter $d$ distributed randomly with constant number density $n$, i.e. the probability density function for finding a cylinder centred at $\boldsymbol{x}$ is uniform. The solid fraction of cylinders is $\alpha=\pi a d / 4$, where

$$
a=n d
$$

is the total frontal area (area exposed to the flow) per unit array volume. For simplicity, we will use $a d$ as a surrogate for the solid fraction. The area containing one cylinder, denoted as the unit cell, is

$$
A_{1}=1 / n=d / a,
$$

so that the mean centre-to-centre spacing between adjacent cylinders in any direction is $s=\sqrt{d / a}$. The mean spacing along any straight transect, e.g. on a line parallel to the direction of flow, is $s_{\|}=1 / a$. This is because the mean number of cylinders per unit length along any such transect, with a finite width $d$, is $n d=a$. The spacing parameters can be normalized as $s^{*}=s / d=1 / \sqrt{a d}$ and $s_{\|}^{*}=s_{\|} / d=1 / \mathrm{ad}$.

It is well-established that when the Reynolds number, $\operatorname{Re}=U d / v$, where $U$ is the velocity upstream of the cylinder and $v$ the kinematic viscosity, is larger than $R e \approx 10$, there exists a wake structure behind each cylinder consisting of a small region of flow reversal and recirculation directly behind the cylinder, and a much larger region downstream of the cylinder in which the velocity is reduced relative to the upstream velocity. The recirculation region, defined specifically as the region over which the time-averaged velocity field exhibits recirculation, will be referred to as the primary wake. The region of velocity defect will be referred to as the secondary wake. This structure is illustrated in figure 1, which shows a unit cell containing a single cylinder with its associated wake. The primary wake extends over a distance referred to as the recirculation length, $l_{r}$, which is $O(d)$ as discussed in $\S 4$. The secondary wake extends a much larger distance known as the attenuation length, also discussed in $\S 4$.

Within the array, the fluid velocity vector is $\boldsymbol{u}$, and the $x$-axis is aligned with the primary direction of fluid flow. The average fluid velocity, $\bar{u}$, is defined by a transverse average across the infinite array and averaged over a time scale sufficient to remove 


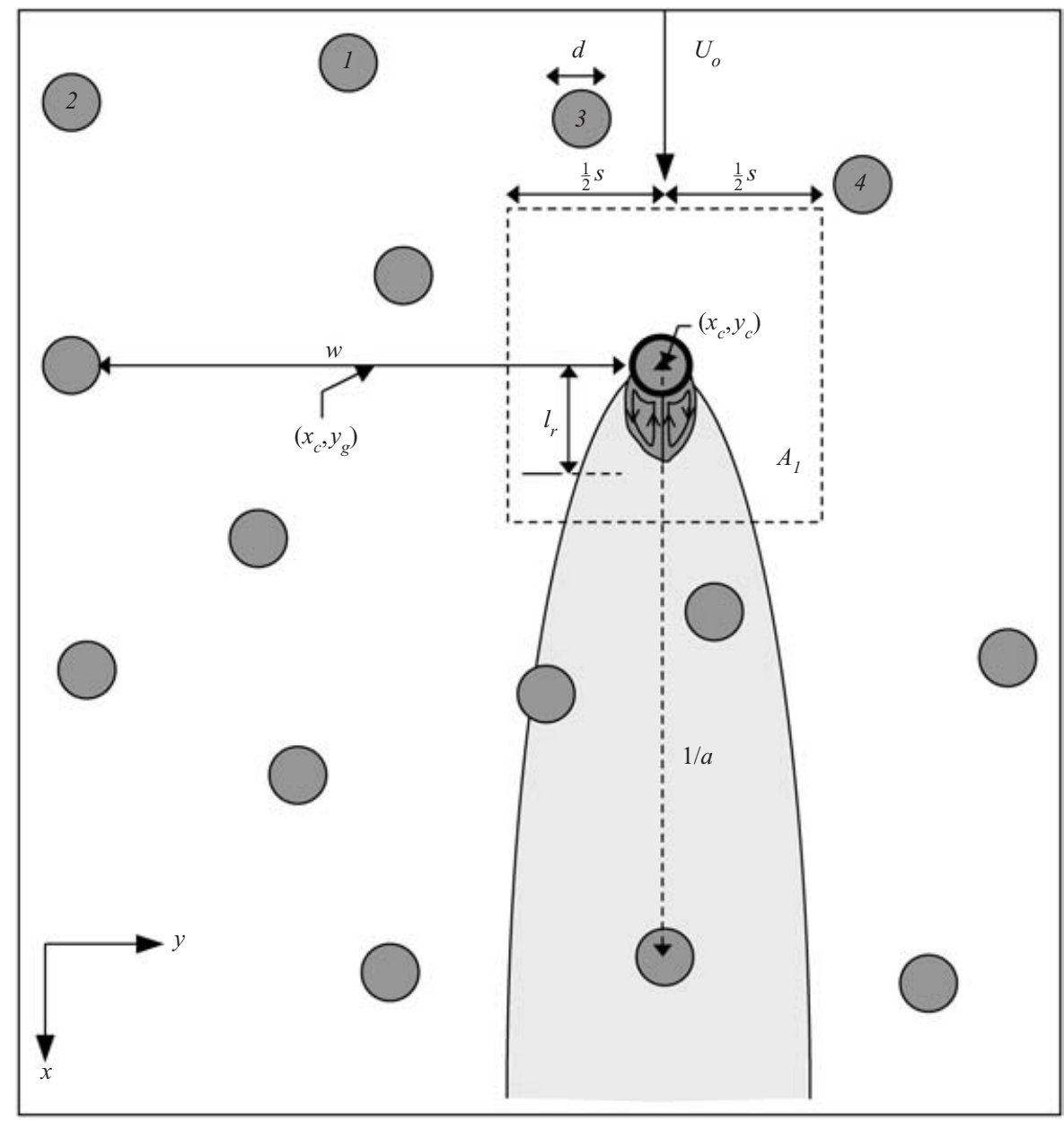

FIgURE 1. An arbitrary array section. The unit cell of area $A_{1}$ is outlined by the dashed box. The fixed cylinder is centred in the unit cell at $\left(x_{c}, y_{c}\right)$ and has associated with it both a primary wake (darker shading) of area $A_{w}$ and a secondary wake (lighter shading). The primary wake, of length $l_{r}$, is the region in which the time-averaged velocity field exhibits recirculation (shown). The secondary wake extends beyond $x=l_{r}$, and decays over the attenuation length scale of $1 / C_{D} a$ according to equation (35). The gap between adjacent cylinders, $w$, admits compensating flow to balance the wake defect. Surrounding cylinders are numbered as in equation (61).

time-dependence,

$$
\bar{u} \boldsymbol{i}=\lim _{t \rightarrow \infty} \lim _{y \rightarrow \infty} \frac{1}{t} \frac{1}{2 y} \int_{0}^{t} \mathrm{~d} t \int_{-y}^{y} \boldsymbol{u} \mathrm{d} y^{\prime} .
$$

Although a time-average is performed in (3), we do not expect the spatially averaged flow to exhibit time-dependence. This is because the time-dependence in the flow is local to each element wake, and thus, in a random array, is distributed randomly with no spatial correlation at scales beyond an individual wake. Even in a periodic array of cylinders, where spatial correlations might be expected, Hill \& Koch (2002) found that for sufficiently large Reynolds number, the fluctuations in drag force are chaotic and uncorrelated at distances beyond the length scale of a unit cell. 
Since $\bar{u}$ represents the average velocity over the entire array, including the solid fraction, it is important to define also the average velocity within the fluid, or in the 'pore space', $U_{o}=\bar{u} /(1-\alpha)$. From the mean fluid velocity, we define the Reynolds number, $R e=U_{o} d / v$, where $v$ is the kinematic fluid viscosity. The array-averaged velocity only yields information about the mean advective mass flux within the array. To obtain a more detailed description, and to predict dispersion, it is necessary to account for the deviations from the array-averaged quantities that occur on the scale of the individual cylinders. Here, we consider both temporal and spatial deviations near each cylinder. We begin with the equations that describe the flow on the cylinder-scale, namely the two-dimensional incompressible Navier-Stokes equations with constant fluid density,

$$
\begin{gathered}
\left(\partial_{t}+\boldsymbol{u} \cdot \nabla\right) \boldsymbol{u}=-\frac{1}{\rho} \nabla p+v \nabla^{2} \boldsymbol{u}, \\
\nabla \cdot \boldsymbol{u}=0 .
\end{gathered}
$$

A general average, whose conditions are as yet unspecified, can then be applied to obtain

$$
\left(\partial_{t}+\langle\boldsymbol{u}\rangle \cdot \nabla\right)\langle\boldsymbol{u}\rangle=-\frac{1}{\rho} \nabla\langle p\rangle+\nu \nabla^{2}\langle\boldsymbol{u}\rangle-\nabla \cdot\left\langle\boldsymbol{u}^{\prime} \boldsymbol{u}^{\prime}\right\rangle,
$$

where the general averaging procedure is denoted by the angular brackets and $\boldsymbol{u}^{\prime}=\boldsymbol{u}-\langle\boldsymbol{u}\rangle$ are fluctuations from the average, the cross-correlations of which yield a net stress term. First, consider the average in (5) to be a transverse cross-sectional average, as in (3), i.e. the array-average. The average velocity is then constant and equal to $U_{o}$, so that the inertial and viscous terms in (5) vanish. However, the presence of the cylinders gives rise to a net resistance force per unit volume, given by the number density of cylinders times the average drag force, $\boldsymbol{F}_{o}$, exerted by the fluid on each cylinder per unit length in the axial direction. The averaged momentum equation is thus

$$
0=-\nabla p_{o}-\frac{a}{d} \boldsymbol{F}_{o},
$$

where the resistance, $\boldsymbol{F}_{o}$, is the average drag force per cylinder due to both surface shear stress and form drag. Specifically, $\boldsymbol{F}_{o}$ arises by evaluating the second and third terms on the right-hand side of (5) around each cylinder, as in Howells (1974) and Hinch (1977).

To describe the deviations from the array-averaged quantities near each cylinder, we take the average in (5) to be a conditional average, conditioned on the presence of a cylinder at a fixed coordinate $\boldsymbol{x}_{1}$. This conditional average is denoted by \langle\rangle$_{1}$ so that the averaged momentum equation is

$$
\left(\partial_{t}+\langle\boldsymbol{u}\rangle_{1} \cdot \nabla\right)\langle\boldsymbol{u}\rangle_{1}=-\frac{1}{\rho} \nabla\langle p\rangle_{1}+v \nabla^{2}\langle\boldsymbol{u}\rangle_{1}-\frac{a}{d} \frac{\langle\boldsymbol{F}\rangle_{1}}{\rho},
$$

where $\langle\boldsymbol{u}\rangle_{1}$ is the velocity field around an average cylinder, i.e. the velocity disturbance created by each cylinder, and $(a / d)\langle\boldsymbol{F}\rangle_{1}$ is the average resistance, exerted by the whole array, opposing the velocity disturbance. The velocity disturbance, $\langle\boldsymbol{u}\rangle_{1}$, describes the flow both in the primary and secondary wake regions behind an average cylinder. Because the velocity is reduced in the wake relative to the array average, i.e. $\langle\boldsymbol{u}\rangle_{1}<U_{o}$, the conditionally averaged drag, $\langle\boldsymbol{F}\rangle_{1}$, is weaker than the array-averaged drag, $\boldsymbol{F}_{o}$. However, the velocity disturbance decays away from the cylinder, so that far from the cylinder the effect of the fixed cylinder is no longer felt and the conditional average is 
simply the array average, $\langle\boldsymbol{u}\rangle_{1}=U_{o}$ and $\langle\boldsymbol{F}\rangle_{1}=\boldsymbol{F}_{o}$. The attenuation of the cylinder disturbance is discussed in greater detail in $\S 4.1$, and (7) is used to derive a component of longitudinal dispersion due to spatial heterogeneity in the flow.

\subsection{Cylinder interactions at high packing density}

The disturbance created by each cylinder depends on the cylinder density and configuration. Interactions between neighbouring cylinders become strong for large $a d$. Much of the analysis of dispersion presented in this paper assumes the existence of a unique and independent wake behind each cylinder (see figure 1). However, when the array is densely packed, neighbouring wakes can affect one another or even coalesce. For example, side-by-side cylinders with a centre-to-centre spacing, $T$, less than $1.2 d$ will act as a single body with a single wake (Zhang \& Zhou 2001). For $1.2<T / d<5$, the two wakes are distinct, but strongly interact. Only for $T / d>5$ are the two wakes independent. Similarly, two in-line cylinders with a centre-to-centre spacing $L / d<1.8$ form a single wake. Distinct in-line wakes do not occur unless $L / d>4$. The above limits for lateral $(T)$ and longitudinal $(L)$ spacing suggest that, in a square array, independent wakes only exist for packing densities up to $a d=0.05$. This limit is consistent with the density at which element drag reduction due to wake interference begins for a square array (Nepf 1999). For a random array, the which average spacing between in-line cylinders, which is $1 / \mathrm{ad}$, is larger than in a square array, for which the in-line spacing is $1 / \sqrt{a d}$. Thus, wake interference should be delayed to slightly larger packing densities. An approximate upper limit for the present theory for random arrays would be $O(0.1)$. While most coastal and bank vegetation is within this limit of stem area density, some freshwater wetlands have densities as high as $a d=0.4$. In $\S 4.3$, we discuss a means of extending the model presented here to these high-density regimes.

\subsection{Scalar transport within the array}

The evolution of scalar concentration over time and space is given by the mass conservation equation

$$
\partial_{t} C=-\nabla \cdot\left(\boldsymbol{u} C-D_{m} \nabla C\right),
$$

where $C(\boldsymbol{x}, t)$ is the scalar concentration and $D_{m}$ is the molecular diffusion constant. We define a Péclet number based on the molecular diffusivity and the array-averaged velocity, $P e=U_{o} d / D_{m}$, which indicates the relative importance of convection and diffusion. To describe the array-scale transport, (8) is first decomposed into temporal and spatial fluctuations at the cylinder scale and then averaged over the array scale. The local concentration and velocity are

$$
\begin{aligned}
& C(\boldsymbol{x}, t)=C_{o}+c^{\prime}(\boldsymbol{x})+c^{\prime \prime}(\boldsymbol{x}, t), \\
& \boldsymbol{u}(\boldsymbol{x}, t)=U_{o}+\boldsymbol{u}^{\prime}(\boldsymbol{x})+\boldsymbol{u}^{\prime \prime}(\boldsymbol{x}, t),
\end{aligned}
$$

where the array averages, denoted with the ' $o$ ' subscript, are defined as in (3); single-primed quantities are temporally averaged spatial fluctuations, and doubleprimed quantities are temporal fluctuations. Introducing these decompositions into the transport equation (8) and taking the array average, denoted by angular brackets, yields

$$
\partial_{t} C_{o}=-\nabla \cdot\left(U_{o} C_{o}-D_{m} \nabla C_{o}+\left\langle\boldsymbol{u}^{\prime} c^{\prime}\right\rangle+\left\langle\boldsymbol{u}^{\prime \prime} c^{\prime \prime}\right\rangle\right) .
$$

The first and second terms on the right-hand side are the fluxes due, respectively, to mean advection and molecular diffusion. The third and fourth terms are correlations 
between the fluctuating velocity and concentration fields. The temporal correlation, $\left\langle\boldsymbol{u}^{\prime \prime} \boldsymbol{c}^{\prime \prime}\right\rangle$, includes the flux due to turbulent transport, denoted $\boldsymbol{J}_{t}$, and an additional contribution from the velocity fluctuations associated with vortex shedding in the primary wake of each cylinder. Specifically, as vortices form, they trap parcels of tracer and hold them back relative to the mean. The random distribution of traps yields a dispersive flux, $\boldsymbol{J}_{v}$. The spatial correlation, $\left\langle\boldsymbol{u}^{\prime} c^{\prime}\right\rangle$, represents a dispersive flux due to the time-averaged spatially random velocity field, which we denote

$$
\boldsymbol{J}_{s}=\left\langle\boldsymbol{u}^{\prime} c^{\prime}\right\rangle
$$

We will show that $\boldsymbol{J}_{s}$ is due to the spatial heterogeneity caused by the superposition of secondary wake defects and can thus be derived by considering the average secondary wake disturbance for a cylinder.

To close the array-scale description we must represent the fluxes $\boldsymbol{J}_{t}, \boldsymbol{J}_{v}$ and $\boldsymbol{J}_{s}$ as functions of the mean velocity and concentration fields $\left(U_{o}\right.$ and $\left.C_{o}\right)$. If the mean concentration field is a slowly varying function of position, i.e. relative to the scale of the fluctuations, the net mass flux will be a linear function of the mean concentration gradient, and the dispersive fluxes obey Fick's law,

$$
\boldsymbol{J}=-D \nabla C_{o},
$$

where the constant of proportionality is the effective dispersion constant. The arrayscale transport equation then becomes

$$
\partial_{t} C_{o}+U_{o} \partial_{x} C_{o}=\left(D_{m}+D_{t}+D_{v}+D_{s}\right) \partial_{x}^{2} C_{o} .
$$

The molecular diffusion constant, $D_{m}$, is a material property and may be assumed known, whereas turbulent diffusion has already been analysed by Nepf (1999), who found that $D_{t}$ increases as $(a d)^{1 / 3}$. The contributions of vortex trapping and the spatially heterogeneous velocity field, $D_{v}$ and $D_{s}$ respectively, will be analysed in the following sections.

We postulate that (14) holds, subject to a posteriori confirmation. The necessary condition for Fickian dispersion, and the existence of an effective dispersion constant, is that the fluctuations in the concentration and velocity fields decay sufficiently fast in time and space. It is shown in $\S 3$ that trapping in the vortex zone leads to Fickian dispersion if the average time a tracer parcel is trapped within the recirculation zone is finite, i.e. the probability distribution function (p.d.f.) of trapping times decays sufficiently fast for long times. That this condition is met can be anticipated from the results of Koch \& Brady (1985), who showed that when tracer is trapped in the boundary layer around a sphere in Stokes flow, molecular diffusion provides an eventual escape mechanism, thus ensuring a finite trapping time and Fickian dispersion within a sphere array. In light of this result, we can at least expect diffusion to provide a mechanism for tracer escape from the recirculation zone. However, in $\S 3$, we will show that escape is controlled by convection, rather than diffusion, leading to a comparatively much shorter trapping time.

The flux due to spatial velocity heterogeneity, $\boldsymbol{J}_{s}$, is Fickian provided the velocity disturbance associated with each cylinder, $\langle\boldsymbol{u}\rangle_{1}$ from (7), decays sufficiently fast away from each cylinder. Such decay is expected based on Brinkman's analysis of viscous porous media, in which resistance from the medium, or Brinkman screening, ensures the decay of the velocity disturbance over a distance known as the Brinkman screening length (Koch \& Brady 1985). Although Brinkman's equations do not hold at moderate Reynolds numbers where inertia is important, we show in $\S 4$ that, for a moderateReynolds-number array, the velocity disturbance associated with each cylinder decays 
over a similar scale, which we call the attenuation length, thus ensuring Fickian dispersion.

Given the velocity disturbance, $\langle\boldsymbol{u}\rangle_{1}$, an explicit expression for the dispersion due to the spatial flux, $\left\langle\boldsymbol{u}^{\prime} c^{\prime}\right\rangle$ can be obtained, and is given in the Appendix. However, this expression is unwieldy, and involves complete knowledge of the unsteady velocity field around a cylinder. In $\S 4$, we approach the problem by a slightly different line of reasoning to obtain an approximate expression for the dispersion constant.

\section{Dispersion from vortex trapping}

The primary wake of an isolated cylinder consists of a recirculation zone with steady closed streamlines for Reynolds numbers above $R e \approx 1$ but less than $R e \approx 40$, at which point a periodic instability sets in. At the point of instability, the recirculation zone begins to oscillate sinusoidally in time, thus opening the closed streamlines and allowing a pathway for fluid transport into and out of the recirculation zone (Gerrard 1978). The rate of entrainment of free-stream fluid into the primary wake and the length of time the entrained fluid remains there is dependent on the Reynolds number. At Reynolds numbers just above the onset of the periodic oscillation, vortex shedding is not observed, and a fluid parcel remains in the primary wake for many oscillation cycles (Gerrard 1978). At a Reynolds number in the range $55<R e<70$, vortex shedding begins, and scalar is released from the primary wake with the shedding vortices. For Reynolds numbers in this range but below $R e \approx 100$, the vorticity-bearing fluid drawn into the wake cavity resides there long enough for its circulation to be cancelled by diffusion of opposite-signed vorticity from the opposite side of the wake. However, above $R e \approx 100$, convection of vorticity dominates diffusion, and the entrained fluid is very quickly removed from the wake cavity, usually on the next shedding cycle (Gerrard 1978). The residence time for tracer particles captured in the primary wake of a flat plate was measured by MacLennan \& Vincent (1982) for Reynolds numbers above $R e=1000$ based on plate width. The residence time, $\tau$, followed an exponential distribution, $\psi(\tau) \propto \exp (-\tau / \bar{\tau})$, where $\bar{\tau}$ is the mean residence time. They observed a strong dependence of $\bar{\tau}$ on $R e$, in accordance with the Reynolds-number-dependence of the vortex-shedding frequency. They also observed that $\bar{\tau}$ was independent of turbulence intensity, suggesting that vortex formation, and not turbulent mixing, is the sole mechanism for trapping and release.

To calculate the dispersion constant due to vortex trapping, consider an ensemble of tracer particles advecting through the array with the mean fluid velocity, $U_{o}$. Each time a particle is entrained into a primary wake, it is trapped and held back from the ensemble for a time $\tau$ taken from the probability density function, $\psi(\tau)$. It thus experiences an excursion of $-U_{o} \tau$ relative to the mean ensemble position. The random distribution of traps as well as the distribution of trapping times leads to a net dispersion of tracer, which is Fickian after each particle in the ensemble has sampled several traps.

The dispersion constant can be calculated from the Lagrangian velocity autocorrelation function, $\overline{u^{\prime}(t) u^{\prime}(t+\tau)}$, where $u^{\prime}(t)=u(t)-U_{o}$, and the overbar represents an average over the ensemble of tracer particles. This function is simple to calculate, since particles in the free stream advect with the mean ensemble, such that $u^{\prime}=0$, while particles residing in traps are stationary, i.e. $u^{\prime}=-U_{o}$. Introducing the conditional probabilities that at time $t$ a particle is inside a trap, $p_{w}(t)$, or is in the free stream, $p_{f}(t)$, the autocorrelation function can be written as the weighted sum of two 
conditional averages,

$$
\overline{u^{\prime}(t) u^{\prime}(t+\tau)}=\left\langle u^{\prime}(t) u^{\prime}(t+\tau)\right\rangle_{w} p_{w}(t)+\left\langle u^{\prime}(t) u^{\prime}(t+\tau)\right\rangle_{f} p_{f}(t),
$$

where $\left\langle u^{\prime}(t) u^{\prime}(t+\tau)\right\rangle_{w}$ and $\left\langle u^{\prime}(t) u^{\prime}(t+\tau)\right\rangle_{f}$ are the correlation functions averaged over the subensembles of, respectively, particles within a trap at time $t$, and particles in the free stream at time $t$. It is easy to see that $\left\langle u^{\prime}(t) u^{\prime}(t+\tau)\right\rangle_{f}=0$ for all $\tau$ since $u^{\prime}(t)=0$ for free-stream particles. Moreover, because the random distribution of cylinders in the array is spatially uniform, the probability of a tracer parcel residing in a trap is a stationary function with respect to the moving particle ensemble. This implies that $p_{w}(t)$ is constant in space and time, and is simply equal to the total proportion of fluid volume occupied by primary wakes, $\epsilon$. The autocorrelation function for the ensemble is thus

$$
\overline{u^{\prime}(t) u^{\prime}(t+\tau)}=\epsilon\left\langle u^{\prime}(t) u^{\prime}(t+\tau)\right\rangle_{w}=\epsilon U_{o}^{2} k(\tau),
$$

where $k(\tau)$ is the probability that a tracer particle in a trap at time $t$ remains there at $t+\tau$. This function is common in statistics, and is known as the survival function,

$$
k(\tau)=1-\int_{0}^{\tau} \psi\left(\tau^{\prime}\right) \mathrm{d} \tau^{\prime} .
$$

The dispersion constant for the trapping process is given by the rate of growth of the ensemble variance (Csanady 1973)

$$
D_{v}=\lim _{t \rightarrow \infty} \frac{1}{2} \frac{\mathrm{d} \sigma^{2}}{\mathrm{~d} t}=\int_{0}^{\infty} \overline{u^{\prime}(t) u^{\prime}(t+\tau)} \mathrm{d} \tau=\int_{0}^{\infty} \epsilon U_{o}^{2} k(\tau) \mathrm{d} \tau=\epsilon U_{o}^{2} \bar{\tau}
$$

where $\bar{\tau}=\int_{0}^{\infty} k(\tau) \mathrm{d} \tau=-\int_{0}^{\infty} \tau(\mathrm{d} k / \mathrm{d} \tau) \mathrm{d} \tau$ is the mean trap duration, i.e. the first moment of the trapping time p.d.f., $\psi(\tau)$.

From (18), the existence of $D_{v}$ requires only that the mean residence time within a primary wake, $\bar{\tau}$, be finite so that the integral in (18) converges. That is, the distribution for trapping times, $\psi(\tau)$, must decay sufficiently fast as $\tau \rightarrow \infty$. The exponential distribution found by MacLennan \& Vincent (1982) certainly satisfies this requirement, but if, for instance, $\psi(\tau) \propto \tau^{-\mu}$ where $\mu \leqslant 2$, then the integral would diverge, and $D_{v}$ would not exist. Such a scenario would result in anomalous (non-Fickian) dispersion (see, e.g. Young 1988; Weeks \& Swinney 1998).

As discussed earlier, in the presence of vortex shedding, convection dominates diffusion, so the residence time $\bar{\tau}$ is expected to be inversely proportional to the frequency of oscillation, $f_{s}=S t U_{o} / d$, where $S t$ is the Strouhal number. Specifically, convection dominates if the time scale for oscillation is much shorter than the time scale for diffusion across the wake, or $S t P e \gg 1$. This is expected for moderate Reynolds numbers and most solutes of interest since $P e=R e S c$, where $S c$ is the Schmidt number of the solute which is typically $O\left(10^{3}\right)$ or higher for most solutes, though it is $O(10)$ for heat.

Confident that convection controls escape from the primary wake at moderate Reynolds number, we can express the mean residence time as $\bar{\tau}=\beta / f_{s}$, where the constant of proportionality $\beta$ is a function of $R e$. Furthermore, since each cylinder in the array has associated with it a primary wake, the total primary wake volume must be proportional to the cylinder density, so we write $\epsilon=\kappa a d$. The constant $\kappa$ is a function of $R e$ since the recirculation length changes considerably with $R e$ (Gerrard 1978). Note that when the cylinder packing density is high enough for multiple wake interactions to occur between adjacent cylinders $(a d \approx 0.1), \kappa$ will show a dependence on the cylinder density and packing configuration as well. 
Substituting the above expressions for $\bar{\tau}$ and $\epsilon$ into (18) an expression for the dispersion constant is obtained:

$$
D_{v}=\frac{\beta \kappa}{S t} a d U_{o} d .
$$

Finally, it must be noted that (19) describes the Fickian limit of vortex trapping, and thus is only valid after the central limit theorem applies, or after each particle has been trapped many times. To determine the time scale necessary to reach the Fickian regime, consider the time scale for a single particle to be trapped exactly once, $\mathfrak{I}$. This time scale is obtained from the volumetric flux of fluid into all primary wake volume, $V_{w}$. This flux is given by $Q_{w}=V_{w} / \bar{\tau}$, as required by continuity. If the total fluid volume in the array is $V_{f}$, then the time scale for all fluid parcels to be trapped exactly once is the turnover time for the entire fluid volume,

$$
\mathfrak{J}=V_{f} / Q_{w}=\frac{\bar{\tau}}{\epsilon}
$$

Note that the inverse of this time scale may be viewed as the trapping frequency. Thus, the Fickian limit is reached asymptotically in the time scale $t \gg \mathfrak{I}$, or

$$
t \gg \frac{\beta}{\kappa S t a d} \frac{d}{U_{o}} .
$$

The time required increases as the array becomes sparser (decreasing $a d$ ). Prior to the Fickian limit, the tracer distribution will exhibit a long upstream tail, corresponding to the fraction of tracer that has been trapped the longest and/or most frequently.

At this point, the issue of re-entrainment of tracer into a single wake should be addressed. It is conceivable that a parcel of tracer may be trapped more than once within the same wake. Results from Duan \& Wiggins (1997) for cylinder wakes at Reynolds numbers of 100 and 190 show that re-entrainment is a rare event. In any case, re-entrainment events do not effect the expression for dispersion, since such events are already reflected in the trapping time distribution, $\psi(\tau)$, and in the average tracer flux, $Q_{w}$.

\subsection{Boundary-layer dispersion}

The vortex-trapping dispersion described in this section is similar to the boundarylayer dispersion associated with trapping in the viscous boundary layer around array elements identified by Koch \& Brady (1985). Both scale in proportion to the number density of trapping sites, which in both cases is proportional to the solid fraction. The primary difference, however, is that tracer escapes the primary wake by convection, whereas diffusion is necessary for boundary-layer escape. As a result, from (19), the vortex-trapping dispersion constant, when normalized by the molecular diffusivity, scales as $D_{v} / D_{m} \sim P e$, whereas boundary-layer dispersion has $P e \log P e$ scaling (Koch \& Brady 1985). Because diffusion controls boundary-layer escape, boundary-layer dispersion operates on a much longer time scale than vortextrapping dispersion. Here, we give simple scaling arguments to compare the magnitude of boundary-layer dispersion to vortex-trapping dispersion in the moderate-to-high Reynolds-number regime.

Around each cylinder, of diameter $d$, is a boundary layer, of width $\delta$, that is defined as the region in which, moving closer to the cylinder surface, diffusion first becomes comparable to convection,

$$
\delta^{2} / D_{m} \sim d / u_{\delta},
$$


where $u_{\delta}$ is the local velocity tangential to the cylinder surface at the outer edge of the boundary layer. We will assume that the boundary layer is close enough to the cylinder surface that the shear stress, $\tau$, throughout the boundary layer can be approximated as constant and equal to its value at the cylinder surface, i.e. the tangential velocity component is approximated as linear in the direction normal to the cylinder surface. This linear approximation of the velocity profile implies that the surface shear stress is related to the boundary-layer velocity by $\tau_{w}=\mu u_{\delta} / \delta$, where $\tau_{w}(\theta), u_{\delta}(\theta)$ and $\delta(\theta)$ all vary with the angular coordinate measured from the front stagnation point of the cylinder. It is common to express the surface stress by a friction coefficient,

$$
c_{f}=\int_{0}^{\pi} \frac{\tau_{w} \sin \theta \mathrm{d} \theta}{\frac{1}{2} \rho U_{o}^{2}}
$$

which has been found for a laminar boundary layer on a circular cylinder $(100<R e<$ $\left.3(10)^{5}\right)$ to be $c_{f}=5.93 / \sqrt{R e}$. From (23), the wall shear stress scales as $\tau_{w} / \rho \sim c_{f} U_{o}^{2}$, and it follows that the local velocity scale is thus

$$
u_{\delta}=\tau_{w} \delta / \mu \sim \rho c_{f} U_{o}^{2} \delta / \mu .
$$

Substituting the velocity scale into (22) yields a scale for the boundary-layer thickness,

$$
\delta / d \sim\left[\frac{1}{c_{f} \operatorname{RePe}}\right]^{1 / 3} .
$$

The time scale for tracer to escape this boundary layer by molecular diffusion is then

$$
T_{d} \sim \frac{\delta^{2}}{D_{m}} \sim P e^{1 / 3}\left(\frac{1}{c_{f} R e}\right)^{2 / 3} \frac{d}{U_{o}} .
$$

Given the scale for the boundary-layer thickness, it is possible to evaluate the accuracy of the linear approximation of the tangential velocity profile within the boundary layer. From the Blasius series solution to the steady two-dimensional boundary-layer equations for a laminar cylinder wake, the linear approximation is reasonable for distances from the cylinder surface of order $y / d \sim 0.1 / \sqrt{R e}$ (Schlichting 1987, figure 10.8). From (25), using the relation $P e=R e S c$ and the experimental value of $c_{f}$ given above, the boundary-layer thickness can be rewritten $\delta / d \sim(1 / \sqrt{R e})[1 / 5.93 S c]^{1 / 3}$. For typical solutes, $S c=O\left(10^{3}\right)$ (though for heat $S c=O(10)$ ), yielding values for the boundary-layer thickness in the range $\delta / d \sim\left(10^{-2}-10^{-1}\right) / \sqrt{R e}$, which is within the range of linearity, i.e. is within the constant stress layer. Thus, the linear approximation is valid for most solutes.

In theory, the contribution from boundary-layer dispersion could be obtained by solving the convection-diffusion equation within the boundary layer, where both convection and diffusion are significant. Such an approach was taken by Koch \& Brady (1985) for spheres in Stokes flow to obtain the $P e \log P e$ scaling for boundary-layer dispersion. However, the velocity disturbance around moderateReynolds-number cylinders does not have a simple analytical expression, as it is characterized by an inertial boundary-layer structure and wake separation, thus precluding a simple solution of the convection-diffusion equation. Alternatively, the boundary-layer dispersion constant could be obtained by the arguments used to obtain the expression for vortex-trapping dispersion. We would need to know the tracer survival distribution within the boundary layers, $k(\tau)$ from (16). A similar method was followed by Young \& Jones (1991) for boundary layers around spheres in Stokes flow using a local expansion of the Stokes stream function. They determined 
that the survival distribution for particles trapped in the boundary layer of a sphere had a $1 / t$ time dependence, thus leading to the $P e \log P e$ scaling for boundary-layer dispersion, and replicating the results of Koch \& Brady (1985). However, lack of an analytical expression for the velocity disturbance within the boundary layer of a moderate-Reynolds-number cylinder makes it difficult to obtain an expression for $k(\tau)$. Thus, to obtain an approximation for the boundary-layer dispersion constant, we use (18) with the mean residence time approximated by the diffusive time scale, $\bar{\tau} \sim T_{d}$, and the boundary-layer volume ratio $\epsilon_{b l} \propto a d \delta / d$ to obtain

$$
D_{b l} \sim \frac{a d}{c_{f} R e} U_{o} d .
$$

This estimate constitutes an upper bound on the dispersion constant because it overestimates the mean residence time within the boundary layer as the diffusive time scale, neglecting the portion of tracer that escapes slowly by convection prior to the diffusive limit. However, (27) is intuitively reasonable, given that when the experimental-value for $c_{f}$ is used, the normalized dispersion constant scales as $D_{b l} / D_{m} \sim a d P e / \sqrt{R e}$, exhibiting the same $1 / \sqrt{R e}$ dependence as the momentum boundary-layer thickness for a circular cylinder at moderate Reynolds numbers (Schlichting 1987). As $R e$ increases, $D_{b l}$ decreases as convection is increasingly more efficient at sweeping tracer away from the boundary layers, diminishing both the residence time within the boundary layers and their thickness. The $P e / \sqrt{R e}$ scaling that we obtain differs from the $P e \log P e$ scaling in Stokes flows. In fact, our result for inertial flows is independent of molecular diffusion. This is because the residence time within the boundary layer grows as $P e^{1 / 3}$, but the boundary-layer thickness decreases as $P e^{-1 / 3}$, so the effects cancel exactly.

With (27), we can compare the contribution from boundary-layer dispersion to that of vortex trapping. Both are proportional to $a d$ and thus grow with the cylinder packing density. From both (19) and (27), the ratio of their magnitudes is

$$
\frac{D_{b l}}{D_{v}} \sim \frac{S t}{\beta \kappa c_{f} R e} .
$$

Since $\beta$ and $\kappa$ are $O(1)$ constants, and $S t$ is typically $O(0.1)$, and using $c_{f}=5.93 / \sqrt{R e}$, it follows that $D_{b l} \ll D_{v}$ over the range of Reynolds numbers for which the cylinder wakes are unsteady. Note also that the time scales at which each process becomes important are very different. The time scale for boundary-layer dispersion to become Fickian is of order

$$
T_{d} / \epsilon_{b l} \sim \frac{P e^{2 / 3}}{\left(c_{f} R e\right)^{1 / 3}} \frac{d}{U_{o} a d} .
$$

This is longer than the time scale for vortex trapping to become a Fickian process (21) by the factor $(\beta / \kappa S t)\left(P e^{2 / 3} /\left(c_{f} R e\right)\right)^{1 / 3}$. In $\S 5$, we discuss typical values for these parameters and show that for most solutes, including that used in our experiments, and for the moderate Reynolds number range we study, the time scale necessary for boundary-layer dispersion to become important is $10^{3}$ times greater than that for vortex trapping. We thus conclude that boundary-layer dispersion plays little role in our study. 


\section{Secondary wake dispersion}

Next, we consider the velocity defect within the secondary wake and show that the superposition of all secondary wakes within the array creates a randomly heterogeneous velocity field, which contributes to dispersion of a passive solute. We first derive a simplified expression for the velocity in the secondary wake, starting from equation (7) for the conditionally averaged velocity field around a cylinder. It is then shown that continuity requires a compensating velocity in the gaps between cylinders to balance the velocity defect in the secondary wakes. The total variance of the random spatial velocity field is determined by superposition of the secondary wake and gap compensation components from all cylinders in the array. The dispersion constant due to the secondary wake is then calculated by considering Lagrangian trajectories through the resulting random velocity field.

\subsection{Statistics of the spatially random field}

To describe the perturbations associated with each wake and gap, we begin with equation (7), the averaged momentum equation conditioned on the presence of a fixed cylinder at $\boldsymbol{x}_{1}$. We are interested in the velocity disturbance from the fixed cylinder over length scales much greater than the immediate $O(d)$ region around it. The turbulent fluctuations in the secondary wake of an isolated cylinder (moderate to high Reynolds number) have a length scale of $O(d)$ or smaller (Schlichting 1987; Rehab, Antonia \& Djenidi 2000). The turbulence scale is thus considerably smaller than the scale over which the secondary wake extends, and so it is reasonable to Reynolds-average (7) and define an effective eddy viscosity, $v_{t}$, to yield

$$
\widetilde{\boldsymbol{u}} \cdot \nabla \widetilde{\boldsymbol{u}}=-\frac{1}{\rho} \nabla \widetilde{p}+\left(v_{t}+v\right) \nabla^{2} \widetilde{\boldsymbol{u}}-\frac{a}{d} \frac{\tilde{\boldsymbol{F}}}{\rho},
$$

with the tilde representing the Reynolds-averaged quantities with respect to the fixed cylinder. It is also useful to define an effective (turbulent) Reynolds number based on the sum of the eddy and molecular viscosities, $R e_{t}=U_{o} d /\left(v_{t}+v\right)$. Moreover, the drag term in (30) can be rewritten in terms of a mean drag coefficient, $C_{D}$, that depends on Reynolds number and the cylinder density, such that $\widetilde{\boldsymbol{F}}=\frac{1}{2} C_{D} d|\tilde{\boldsymbol{u}}| \tilde{\boldsymbol{u}}$. It should be noted that the average value of $C_{D}$ in the array may vary considerably from that for an isolated cylinder (see e.g. Koch \& Ladd 1997; Ayaz \& Pedley 1999; Nepf 1999).

It is important to have an estimate for the eddy viscosity, $v_{t}$. The magnitude of $R e_{t}$ has been obtained for a number of experimental scenarios. Hinze (1975) gives $R e_{t} \approx 65$ for an isolated cylinder, whereas Schlichting (1987) gives a value of approximately 45 . Fitting wake velocity defect profiles given in Kovasznay (1949) and Zavistoski (1994), we have estimated a value of $R e_{t} \approx 35$ in the range from $R e=56$ to $R e=770$. These values suggest that over the Reynolds number range of interest to us, $R e \approx 50$ to $R e \approx 1000$, the value of the eddy viscosity is in the range $v_{t} \approx[1 /(35-65)] U_{o} d$, so that the relative importance of fluid viscosity and turbulent viscosity ranges from $v / v_{t}=O(1)$ to $v / v_{t}=O(0.1)$. This estimate for $v_{t}$ is necessary in order to predict secondary-wake dispersion, which is derived in the following.

To calculate the secondary-wake defect, we are interested in the velocity disturbance, $u_{w}^{\prime}$, only in a region far from the primary wake, where $u_{w}^{\prime} / U_{o} \ll 1$. The conditionally averaged velocity from (30) is decomposed into the array average and the small perturbations, namely, $\widetilde{\boldsymbol{u}}=\left(U_{o}+u_{w}^{\prime}\right) \boldsymbol{i}+v_{w}^{\prime} \boldsymbol{j}$. In addition, for inertial flows, we expect the velocity disturbance to have a thin wake structure, such that the transverse extent, $b$, is small compared with the longitudinal scale, $L$, i.e. $b / L \ll 1$. The relevant variables are thus rescaled by introducing non-dimensional variables denoted with an asterisk, 
namely $x=L x^{*}, y=b y^{*}, u_{w}^{\prime}=q u^{*}, v_{w}^{\prime}=(q b / L) v^{*}$ and $\widetilde{p}=P p^{*}$, where $P$ is the pressure scale to be determined and $q$ is the velocity scale for the longitudinal perturbation $u_{w}^{\prime}$, so that $q \ll U_{o}$. Upon substitution into (30), and after neglecting $O\left(\left(q / U_{o}\right)(b / L)^{2}\right)$ inertial and drag terms and the $O\left(\left(1 / R e_{t}\right)(d / L)(b / L)^{2}\right)$ longitudinal viscous term, we obtain for the longitudinal momentum equation

$$
\left(\frac{b}{L}\right)^{2} u_{x^{*}}^{*}=\frac{-P}{\rho q U_{o}}\left(\frac{b}{L}\right)^{2} p_{x^{*}}^{*}+\frac{1}{R e_{t}} \frac{d}{L} u_{y^{*} y^{*}}^{*}-\frac{1}{2} a L C_{D}\left(\frac{b}{L}\right)^{2}\left(\frac{U_{o}}{q}+2 u^{*}\right) .
$$

Since the inertial term on the left-hand side must be significant, and thus comparable to the pressure term, the pressure scale must be $P / \rho q U_{o}=O(1)$. Additionally, because we are interested in length scales far from the cylinder, say $L \sim s=d / \sqrt{a d}$, by balancing the viscous and inertial terms we deduce $(b / L)^{2}=O\left(\sqrt{a d} / R e_{t}\right)$, which with the values for $R e_{t}$ cited above, affirms the hypothesis that $(b / L)^{2} \ll 1$.

After neglecting terms of $O\left(\left(q / U_{o}\right)(b / L)^{2}\right)$ and smaller, the transverse momentum equation becomes

$$
\left(\frac{b}{L}\right)^{2} v_{x^{*}}^{*}=\frac{-P}{\rho q U_{o}} p_{y^{*}}^{*}+\frac{1}{R e_{t}} \frac{d}{L} v_{y^{*} y^{*}}^{*}-\frac{1}{2} a L C_{D}\left(\frac{b}{L}\right)^{2} .
$$

Because $P / \rho q U_{o}=O(1)$, and the inertial, viscous and drag terms are $O\left(b^{2} / L^{2}\right) \ll 1$ or smaller, we must deduce that the transverse pressure gradient, $p_{y^{*}}^{*}$, is negligible, such that the pressure disturbance in the wake is approximately equal to the background pressure outside the wake, $\widetilde{p} \approx p_{o}$, just as in thin boundary-layer theory. This implies that the pressure disturbance caused by the cylinder is localized, and that away from the cylinder the pressure field is simply the mean field obtained by the zeroth-order momentum balance (6). By this argument, we would expect to find a Green's function for the velocity disturbance arising from the point drag force at the cylinder. Indeed, after approximating the pressure by the mean and returning to dimensional variables, (31) becomes

$$
\partial_{x} u_{w}^{\prime}=\frac{v_{t}+v}{U_{o}} \partial_{y y} u_{w}^{\prime}-C_{D} a u_{w}^{\prime},
$$

which is in the form of a diffusion equation with a decay term due to array drag. The comparison between (33) and the well-known Brinkman equation for Stokes flow in porous media should be noted. As discussed in Howells (1974), the Brinkman equation can be obtained from (7) by neglecting inertial terms. From the Brinkman equation, a Green's function for the velocity disturbance is obtained, which is the Stokes solution around an isolated cylinder modified by the net drag force from the array. The analysis here is similar in spirit, but the importance of inertia and the subsequent wake approximation which allows pressure to be treated as the array average, results in a different, diffusion-type Green's function for the velocity disturbance.

The velocity disturbance is a delta function at the cylinder origin, $\left(x_{c}, y_{c}\right)=(0,0)$, with strength determined by the average drag per cylinder, $\widetilde{\boldsymbol{F}}$. The disturbance satisfies the boundary conditions

$$
u_{w}^{\prime}=0, \quad y= \pm \infty,
$$

which, with the drag condition, leads to

$$
u_{w}^{\prime}\left(x^{*}, y^{*}\right)=-\frac{C_{D} U_{o} \sqrt{R e_{t}}}{4 \sqrt{\pi x^{*}}} \exp \left(-R e_{t} y^{* 2} / 4 x^{*}\right) \exp \left(-C_{D} a d x^{*}\right),
$$


where the position coordinates have been non-dimensionalized, $x^{*}=x / d$ and $y^{*}=$ $y / d$. From (35), it is apparent that the velocity perturbation decays exponentially by array drag over the scale $\left(C_{D} a\right)^{-1}$, which we will call the attenuation length. The attenuation length, much like the Brinkman screening length in a Brinkman porous medium (Howells 1974), gives the distance from a given cylinder at which the disturbance due to that cylinder is no longer felt by the fluid.

For distances much less than the attenuation length, but still outside the primary wake, $l_{r} \ll x \ll\left(C_{D} a\right)^{-1}$, the disturbance given by (35) has the appearance of a typical solution to the one-dimensional diffusion equation, with $x$ playing the role of time. Such diffusive scaling for inertial wake flows is commonly assumed in the far wake, with ample experimental support (see Schlichting 1987, for example). Data from Paranthoen et al. (1999) for an isolated cylinder suggest that for a wide range of Reynolds numbers, diffusive scaling also holds closer to the cylinder if the origin of the momentum sink is offset to the end of the recirculation zone (see figure 1). With this offset, diffusive scaling is evident in the wake velocity profiles of Paranthoen et al. for distances greater than $O(d)$ downstream of the recirculation zone, as shown by figure 2 . We denote the distance between the end of the recirculation zone and the point at which diffusive scaling holds as $x_{o}=O(d)$.

In scaling the momentum equation, we replaced the conditionally averaged pressure field by the array-averaged pressure field. We now consider the small error associated with this approximation. While the pressure disturbance due to a fixed cylinder is localized near the cylinder, a small portion is distributed through the gap between laterally adjacent cylinders. This slightly elevated pressure is necessary to drive flow through the gap to compensate for the lost mass flux in the cylinder wake. The gap width is $O(1 / a d)$, so the velocity compensation is distributed over a much larger width than the point drag force that is responsible for the wake defect. Because of this scale separation, it is advantageous to separate the Green's function for the secondary wake velocity defect from the much more distributed background velocity compensation in the gaps. By integration of (35), the total mass flux deficit associated with each cylinder is

$$
M=\frac{1}{2} \rho C_{D} U_{o} d .
$$

Mass conservation requires an equivalent compensating flow distributed between elements,

$$
u_{g}^{\prime}=\frac{M}{\rho w}=\frac{C_{D} U_{o} a d}{2(1-a d)},
$$

where $w=s_{\|}-d$ is the average gap spacing between laterally adjacent cylinders (see figure 1). Applying the Bernoulli equation across the gap, valid in the inviscid limit $\left(\operatorname{Re}_{t} s / d \gg 1\right)$, the perturbation pressure drop is

$$
\Delta p^{\prime}=\frac{1}{2} \rho u_{g}^{\prime 2}=\frac{1}{8} \rho\left[\frac{C_{D} U_{o} a d}{(1-a d)}\right]^{2},
$$

distributed over a streamwise length scale $O(s=d / \sqrt{a d})$. Comparing the perturbation to the mean gradient, which balances the mean array drag by (6), i.e. $\partial_{x} p_{o}=$ $-\rho C_{D} a U_{o}^{2} / 2$, gives

$$
\frac{d_{x} p^{\prime}}{d_{x} p_{o}} \sim \frac{1}{4} C_{D} \frac{a d^{3 / 2}}{(1-a d)^{2}} .
$$

For small $a d, d_{x} p^{\prime} \ll d_{x} p_{o}$, as assumed to obtain (33). 


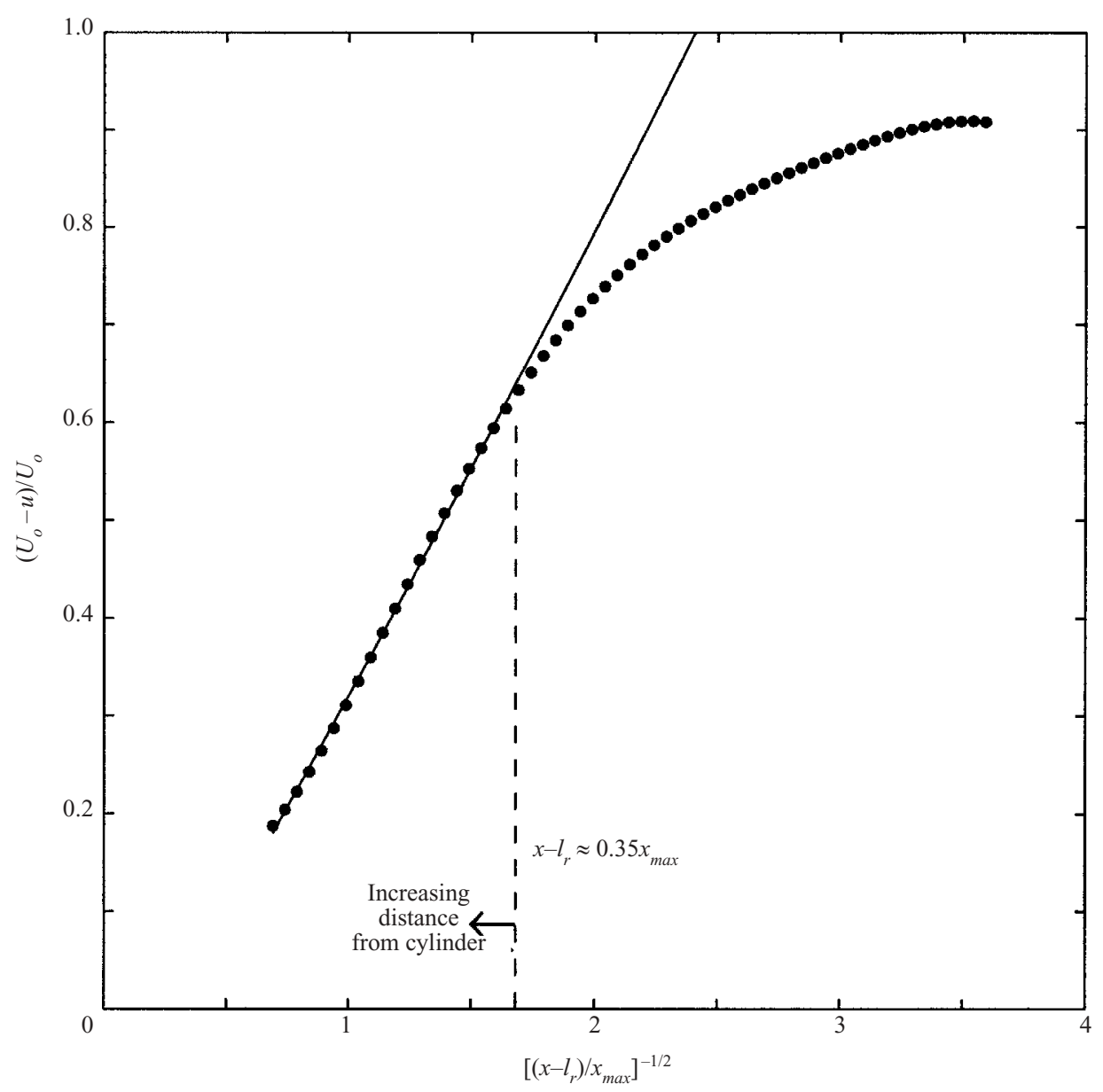

Figure 2. Centreline velocity defect, $\left(U_{o}-u\right) / U_{o}$, for several wake profiles in the range $45<R e<80$ taken from figure 18 in Paranthoen et al. (1999). Their data, which line up remarkably well along a single line in figure 18, were fitted to a fourth-order polynomial to generate the points shown here (solid markers). In Paranthoen et al., the velocity defect is plotted as a function of the renormalized streamwise coordinate, $\left(x-l_{r}\right) / x_{\max }$, where $l_{r}$ is the length of the recirculation zone, and $x_{\max }$ is an Re-dependent constant, $x_{\max }=O(d)$. Here, we plot the defect as a function of $\left[\left(x-l_{r}\right) / x_{\text {max }}\right]^{-1 / 2}$. The linear fit (solid line) suggests that, for an isolated cylinder, the velocity defect decays like $u_{w}^{\prime} \propto 1 / \sqrt{\left(x-l_{r}\right)}$ in the range $x \gtrsim 0.35 x_{\text {max }}+l_{r}$, i.e. the growth of the wake is diffusive, consistent with the theory (35) for $x \ll 1 / C_{D} a$. Note that increasing distance from the cylinder corresponds to decreasing $\left[\left(x-l_{r}\right) / x_{\max }\right]^{-1 / 2}$. This Reynolds number range spans significant changes in near-wake behaviour (Gerrard 1978), yet diffusive scaling holds throughout this range, suggesting that this scaling is robust, and probably extends to higher Reynolds numbers.

To find the evolution of the velocity perturbation, $u_{g}^{\prime}$, we treat the compensating flux as a transverse line source of momentum. Following the same procedure used to derive $u_{w}^{\prime}$, the velocity field is written as the sum of the mean and the gap perturbation and substituted into (30). We linearize, as before, and neglect transverse diffusion of momentum in the gap in order to obtain an upper bound on $u_{g}^{\prime}$. Then

$$
\partial_{x^{*}} u_{g}^{\prime}=-C_{D} a d u_{g}^{\prime},
$$


i.e. owing $u_{g}^{\prime}$ decays to array drag only. With the initial condition

$$
\begin{aligned}
& \left.u_{g}^{\prime}\right|_{x^{*}=0}=\frac{U_{o} C_{D} a d}{2(1-a d)}, \\
& u_{g}^{\prime}(x)=\frac{U_{o} C_{D} a d}{2(1-a d)} \exp \left(-C_{D} a d x^{*}\right), \quad\left|y-y_{g}\right| \leqslant \frac{1}{2} w, \\
& u_{g}^{\prime}(x)=0, \quad\left|y-y_{g}\right|>\frac{1}{2} w,
\end{aligned}
$$

where $y_{g}$ is the mid-width coordinate of the gap associated with each cylinder and the cylinder centre is taken to be $x=0$ (see figure 1). Note that $w$ is a random function, i.e. the gap associated with each cylinder varies randomly with the locations of adjacent cylinders. However, this higher-order effect will be neglected since it will be shown that for the array densities that we address, the gap contribution to the velocity variance is small compared with the wake contribution, thus $w$ is taken to be a constant mean gap width.

To derive the statistics of the random velocity field, we will write the spatial deviation from the mean velocity, $u^{\prime}(x, y)=\tilde{u}(x, y)-U_{o}$, as a superposition of velocity perturbations due to upstream wake defect and gap contributions. Superposition is valid for points outside the primary wake of a fixed cylinder, because here, where the velocity defect is small compared with the mean flow, the momentum equations governing the evolution of the wake and gap velocity perturbations, (33) and (40), are linear. Thus, cylinders and gaps can be viewed as, respectively, point sinks and sources of momentum, whose associated velocity perturbations evolve according to linear diffusion equations. Moreover, the superposition of two or more simple shear layers to describe a complex shear layer was previously described by Bradshaw, Dean \& McEligot (1973), and validated experimentally by Zhou et al. (2000) for complex wakes formed from two or more cylinders. In an area $A$ extending far upstream and in either transverse direction of a point $(x, y)$ there are $N=A a / d$ cylinders which contribute to the total velocity fluctuation at $(x, y)$. Thus, the total fluctuation is given by superposition:

$$
u^{\prime}(x, y)=\sum_{i=1}^{N} u_{w, i}^{\prime}\left(x_{i}^{*}, y_{i}^{*}\right)+\sum_{i=1}^{N} u_{g, i}^{\prime}\left(x_{i}^{*}, y_{g, i}^{*}\right),
$$

where $u_{w, i}^{\prime}$ and $u_{g, i}^{\prime}$ represent, respectively, the wake and gap contributions. $\left(x_{i}^{*}, y_{i}^{*}\right)$ is the normalized distance to the $i$ th cylinder and $\left(x_{i}^{*}, y_{g, i}^{*}\right)$ is the normalized distance to the centre of the gap associated with the $i$ th cylinder.

From the disturbance fields, $u_{w}^{\prime}$ and $u_{g}^{\prime}$, the spatial statistics of the random velocity field, $\tilde{u}(x, y)$, can be constructed from the superposition given by (43). Since upstream cylinders contributing to $u^{\prime}(x, y)$ are randomly distributed, (43) is a sum of independent identically distributed random variables, which is the mathematical formulation of a random walk, where $u_{w, i}^{\prime}\left(x_{i}^{*}, y_{i}^{*}\right)$ and $u_{g, i}^{\prime}\left(x_{i}^{*}, y_{g, i}^{*}\right)$ play the role of the single step displacements in the walk, and $u^{\prime}(x, y)$ is the net displacement after $n$ random steps.

We define $P_{u^{\prime}}\left(u^{\prime}\right)$ as the probability density function (p.d.f.) for $u^{\prime}(x, y)$, and $p_{u_{w}^{\prime}}\left(u_{w}^{\prime}\right)$ and $p_{u_{g}^{\prime}}\left(u_{g}^{\prime}\right)$ as the p.d.f. for $u_{w, i}^{\prime}(x, y)$ and $u_{g, i}^{\prime}(x, y)$, respectively. We seek the mean and variance of $P_{u^{\prime}}\left(u^{\prime}\right),\left\langle u^{\prime}\right\rangle$ and $\sigma_{u}^{2}$, from which the coefficient of dispersion can be obtained. Following random walk formalism (see Hughes 1995, for example), the 
moments of $P_{u^{\prime}}\left(u^{\prime}\right)$ are given in terms of the moments of $p_{u_{w}^{\prime}}\left(u_{w}^{\prime}\right)$ and $p_{u_{g}^{\prime}}\left(u_{g}^{\prime}\right)$ :

$$
\begin{gathered}
\left\langle u^{\prime}\right\rangle=N\left[\left\langle u_{w, i}^{\prime}\right\rangle+\left\langle u_{g, i}^{\prime}\right\rangle\right], \\
\sigma_{u}^{2} \equiv\left\langle u^{\prime 2}\right\rangle-\left\langle u^{\prime}\right\rangle^{2}=N\left[\left\langle u_{w, i}^{\prime 2}\right\rangle-\left\langle u_{w, i}^{\prime}\right\rangle^{2}+\left\langle u_{g, i}^{\prime 2}\right\rangle-\left\langle u_{g, i}^{\prime}\right\rangle^{2}\right],
\end{gathered}
$$

where, in general, $\left\langle g^{n}\right\rangle \equiv \int_{-\infty}^{\infty} g^{n} p(g) \mathrm{d} g$ is the $n$th moment of the density $p(g)$ and $g$ represents either $u_{w, i}^{\prime}$ or $u_{g, i}^{\prime}$. This integral can be rewritten more simply as

$$
\left\langle g^{n}\right\rangle=\lim _{A \rightarrow \infty} \iint_{A} g^{n}(x, y) P_{c}(x, y) \mathrm{d} A,
$$

where $P_{c}(x, y)=a / N d$ is the two-dimensional p.d.f. for the location of the $i$ th cylinder. This is easily verified by setting $n \equiv 0$, so that the zeroth moment of both $p_{u_{w}^{\prime}}$ and $p_{u_{g}^{\prime}}$ is $\iint_{A} a / N d \mathrm{~d} x \mathrm{~d} y=1$, proving that $P_{c}(x, y)$ is properly normalized. We can choose the area $A$ arbitrarily large to include all contributing upstream cylinders, yielding from (45)

$$
\begin{aligned}
& \left\langle u_{w, i}^{\prime n}\right\rangle=\int_{-\infty}^{\infty} \int_{x_{o}}^{\infty} u_{w}^{\prime n}(x, y) P_{c}(x, y) \mathrm{d} y \mathrm{~d} x, \\
& \left\langle u_{g, i}^{\prime n}\right\rangle=\int_{-\infty}^{\infty} \int_{0}^{\infty} u_{g}^{\prime n}(x, y) P_{c}(x, y) \mathrm{d} y \mathrm{~d} x,
\end{aligned}
$$

where the expressions for the wake and gap perturbations, respectively (35) and (41), are used. Also, note that the $O(d)$ distance, $x_{o}$, has been used in the limits of integration to eliminate contributions from primary-wake regions, in which diffusive scaling of the wake perturbation is invalid, and whose contribution to dispersion has already been characterized by vortex-trapping dispersion. Evaluating (45) yields the means if $n=1$,

$$
\left\langle u_{g, i}^{\prime}\right\rangle=-\left\langle u_{w, i}^{\prime}\right\rangle=\frac{1}{2} U_{o},
$$

which satisfies mass conservation in the array since $\langle u(x, y)\rangle \equiv U_{o}=U_{o}+\left\langle u_{w, i}^{\prime}\right\rangle+\left\langle u_{g, i}^{\prime}\right\rangle$. Setting $n=2$ yields, respectively, the variances due to wake and gap contributions,

$$
\begin{gathered}
\sigma_{w}^{2}=\frac{U_{o}^{2}}{16 \sqrt{\pi}} \Gamma\left(\frac{1}{2}, 2 x_{o}^{*} C_{D} a d\right) \sqrt{C_{D}^{3} a d R e_{t}}-\frac{1}{N}\left\langle u^{\prime}\right\rangle^{2}, \\
\sigma_{g}^{2}=\frac{U_{o}^{2} C_{D} a d}{8(1-a d)}-\frac{1}{N}\left\langle u_{g}^{\prime}\right\rangle^{2},
\end{gathered}
$$

where $\Gamma\left(1 / 2,2 x_{o}^{*} C_{D} a d\right)$ is the incomplete gamma function,

$$
\Gamma\left(\frac{1}{2}, 2 x_{o}^{*} C_{D} a d\right)=\int_{2 x_{o}^{*} C_{D} a d}^{\infty}(1 / \sqrt{\tau}) \mathrm{e}^{-\tau} \mathrm{d} \tau .
$$

Since $x_{o}^{*}=x_{o} / d \approx 1,2 x_{o}^{*} C_{D} a d \ll 1$ for sparse arrays, and thus the Taylor expansion of $\Gamma\left(1 / 2,2 x_{o}^{*} C_{D} a d\right)$ can be used to define the function

$\gamma \equiv \frac{1}{\sqrt{\pi}} \Gamma\left(\frac{1}{2}, 2 x_{o}^{*} C_{D} a d\right)=1-\frac{2}{\sqrt{\pi}} \sqrt{2 x_{o}^{*} C_{D} a d}+\frac{2}{3 \sqrt{\pi}}\left(2 x_{o}^{*} C_{D} a d\right)^{3 / 2}+O\left(a d^{5 / 2}\right)$.

Making use of this notation, and recognizing that the second terms in both $(49 a)$ 
and (49b) vanish as $A \rightarrow \infty$ and thus $N \rightarrow \infty$, we finally obtain

$$
\begin{gathered}
\sigma_{w}^{2}=\left\langle u_{w, i}^{\prime 2}\right\rangle-\left\langle u_{w, i}^{\prime}\right\rangle^{2}=\frac{U_{o}^{2}}{16} \gamma \sqrt{C_{D}^{3} a d R e_{t}}, \\
\sigma_{g}^{2}=\left\langle u_{g, i}^{\prime 2}\right\rangle-\left\langle u_{g, i}^{\prime}\right\rangle^{2}=\frac{U_{o}^{2} C_{D} a d}{8(1-a d)} .
\end{gathered}
$$

The total variance of the spatially random velocity field is the sum of the wake and gap variances, $\sigma_{u}^{2}=\sigma_{w}^{2}+\sigma_{g}^{2}$. However, note that $\sigma_{u_{g}^{\prime}}^{2} / \sigma_{u_{w}^{\prime}}^{2} \sim \sqrt{a d / \operatorname{Re}_{t} C_{D}}$, so that for sparse arrays $(a d \lesssim 0.1)$ and $R e_{t} \geqslant O(10)$, the gap contribution is small $\sigma_{u_{g}^{\prime}}^{2} / \sigma_{u_{w}^{\prime}}^{2} \ll 1$. In this case, the wake defects contribute most of the variance, and we can make the approximation

$$
\sigma_{u}^{* 2}=\sigma_{u}^{2} / U_{o}^{2} \approx \sigma_{w}^{2} / U_{o}^{2}
$$

in which the variances have been normalized using the mean velocity. Specifically, in a sparse array, the wake provides a more concentrated velocity perturbation, a point momentum sink, than the gap compensation which is distributed over an $O(1 / \mathrm{ad})$ width. For dense arrays, however, the variance contributed by the gap compensation becomes significant, as fluid is forced through the gaps with a significant pressure drop. It is then no longer acceptable to neglect the gap pressure perturbation, as was done on the basis of (39) to obtain (33). Thus for high packing density, the theory leading to $(51 a)$ is no longer valid, as noted already in $\S 2.1$.

\subsection{Secondary wake dispersion constant}

We now use the variance of the velocity field to calculate the dispersion due to secondary wakes. Specifically, the dispersion coefficient, $D_{s}$, will be determined by estimating the Lagrangian velocity correlations of passive particles advecting through the unit cell, which is shown as a dashed square in figure 1. We assume that tracer particles are distributed throughout the array with uniform concentration $C$, so the constant flux of tracer particles into the unit cell is

$$
\mathrm{d} N / \mathrm{d} t=C U s .
$$

We will follow a discrete packet of particles entering in a discrete time $\mathrm{d} t=\mathrm{d} x / U$, and thus numbering

$$
N=C s \mathrm{~d} x \text {. }
$$

The velocity autocorrelation function averaged over all trajectories is

$$
\mathscr{R}(\tau) \equiv \overline{u_{i}^{\prime}(0) u_{i}^{\prime}(\tau)}=\lim _{N \rightarrow \infty} \frac{1}{N} \sum_{i=1}^{N} u_{i}^{\prime}(0) u_{i}^{\prime}(\tau),
$$

where $u_{i}^{\prime}(\tau)$ is the instantaneous velocity of the $i$ th particle at time $\tau$ along its trajectory and the overbar represents the average over all trajectories. Using the expression for $N$, (54), and integrating the velocity correlation function over the range of initial transverse particle locations $\zeta$ within the unit cell gives

$$
\mathscr{R}(\tau)=\frac{1}{C s \mathrm{~d} x} \int_{-s^{*} / 2}^{s^{*} / 2} u^{\prime}(0) u^{\prime}(\tau) C \mathrm{~d} x \mathrm{~d} \zeta=\sqrt{a d} \int_{-s^{*} / 2}^{s^{*} / 2} u^{\prime}(0) u^{\prime}(\tau) \mathrm{d} \zeta .
$$

Given $\mathscr{R}(\tau), D_{s}$ follows directly

$$
D_{s}=\int_{0}^{\infty} \mathscr{R}(\tau) \mathrm{d} \tau .
$$


We can approximate $\mathscr{R}(\tau)$ using the spatial velocity statistics defined previously. Specifically, $\mathscr{R}(\tau)$ is estimated by following an ensemble of particles advecting through the time-averaged velocity field and assuming they execute random motion in the transverse direction due to turbulence and/or Brownian motion, with an effective transverse diffusion constant $D_{y}$. We also define an effective Schmidt number based on the particle diffusion constant and the effective turbulent viscosity, $S c_{t}=\left(v+v_{t}\right) / D_{y}$.

If the time scale for transverse diffusion across the unit cell, i.e. across width $s$, is much longer than the time scale for advection over the attenuation length, $1 / C_{D} a$, then the net dispersion caused by the velocity disturbance for each wake will be mechanical, i.e. determined by advection, that is if

$$
\frac{\left(s^{2} / D_{y}\right)}{1 / U_{o} C_{D} a}=C_{D} R e_{t} S c_{t} \gg 1 .
$$

For the moderate-Reynolds-number flows we examine, this relation is expected to hold because in turbulent flows, $S c_{t} \approx 1$, for mass (true also for the turbulent Prandtl number for heat), $C_{D}=O(1)$, and $R e_{t} \gg 1$, as discussed in $\S 4.1$. In the laminar regime, for which transverse diffusion is Brownian, $D_{y}=D_{m}, S c_{t} \gg 1$ for typical solutes. Thus, from (58), dispersion should be mechanical for moderate Reynolds number, so the Lagrangian particle velocity in each wake, $u^{\prime}(\tau)$ can be estimated by advection and diffusion of the ensemble through the wake defect (35). Equations (56) and (57) thus yield

$$
\begin{aligned}
D_{s}=\sqrt{a d} \int_{0}^{\infty} \frac{\mathrm{d} x^{*}}{u\left(x^{*}+x_{o}^{*}, y^{*}\right)} & \int_{-s^{*} / 2}^{s^{*} / 2} \mathrm{~d} \xi u_{w}^{\prime}\left(x_{o}^{*}\right) \\
\times & \int_{-s^{*} / 2}^{s^{*} / 2} \mathrm{~d} y^{*} p\left(x^{*}+x_{o}^{*}, y^{*} \mid x_{o}^{*}, \xi\right) u_{w}^{\prime}\left(x^{*}+x_{o}^{*}, y^{*}\right),
\end{aligned}
$$

where $p\left(x^{*}+x_{o}^{*}, y^{*} \mid x_{o}^{*}, \xi\right)$ is the Gaussian propagator for the transverse diffusive motion

$$
p\left(x^{*}+x_{o}^{*}, y^{*} \mid x_{o}^{*}, \xi\right)=\sqrt{\frac{R e_{t} S c_{t}}{4 \pi x^{*}}} \exp \left[-\frac{R e_{t} S c_{t}}{4 x^{*}}\left(y^{*}-\xi\right)^{2}\right] .
$$

Since within the secondary wake, $u_{w}^{\prime} \ll U_{o}$, the time integration in (59) is converted to spatial integration using the identity

$$
\mathrm{d} t=\frac{\mathrm{d} x}{u(x(t))}=\frac{\mathrm{d} x}{U_{o}}+O\left(u_{w}^{\prime} / U_{o}\right)
$$

similar to Taylor's frozen turbulence theorem. In evaluating (59), we will consider only the leading-order term, $\mathrm{d} x / U_{o}$.

Before evaluating (59), it is important to determine the contribution from cylinders outside the unit cell to the autocorrelation function for the cylinder wake within the unit cell. The integral in (59) contains the spatial correlation, $\left\langle u_{w}^{\prime}\left(x_{o}\right) u_{w}^{\prime}(x)\right\rangle$, within the unit cell. By superposition, as in (43), $u_{w}^{\prime}(x)$ is composed of contributions from the cylinder within the unit cell, which we denote $u_{1}^{\prime}(x)$, and from those outside, $u_{i}^{\prime}(x), i=2, \ldots, n$, so that

$$
\left\langle u_{w}^{\prime}\left(x_{o}\right) u_{w}^{\prime}(x)\right\rangle=\left\langle\left(u_{1}^{\prime}\left(x_{o}\right)+u_{2}^{\prime}\left(x_{o}\right)+\ldots u_{n}^{\prime}\left(x_{o}\right)\right)\left(u_{1}^{\prime}(x)+u_{2}^{\prime}(x)+\ldots u_{n}^{\prime}(x)\right)\right\rangle .
$$

Refer to figure 1 for an example configuration of contributing cylinders outside the unit cell. All cross-terms in (61) involving the primary wake, $\left\langle u_{1}^{\prime}\left(x_{o}\right) u_{i}^{\prime}(x)\right\rangle$ and $\left\langle u_{i}^{\prime}\left(x_{o}\right) u_{1}^{\prime}(x)\right\rangle$ for $i \neq 1$, are zero since $u_{1}^{\prime}(x)$ is deterministic within the unit cell and 
can be taken outside the average, leaving $\left\langle u_{i}^{\prime}(x)\right\rangle=0$. Autocorrelation terms like $\left\langle u_{i}^{\prime}\left(x_{o}\right) u_{i}^{\prime}(x)\right\rangle$ are estimated simply by the velocity defect for a single cylinder (35). This leaves higher-order cross-terms: $\left\langle u_{i}^{\prime}\left(x_{o}\right) u_{j}^{\prime}(x)\right\rangle, i \neq j, i, j>1$, which represent the wake interactions, and which cannot be accounted for without extending the present framework to a higher-order analysis. We can estimate the error incurred by neglecting these terms. The maximum interaction is obtained for in-line cylinders, and at the distance $O(s)$ from the fixed cylinder, at which

$$
\frac{\left\langle u_{i}^{\prime}\left(x_{o}\right) u_{j}^{\prime}(x)\right\rangle}{\left\langle u_{1}^{\prime}\left(x_{o}\right) u_{1}^{\prime}(x)\right\rangle} \approx \frac{a d^{1 / 4}}{\sqrt{3}} \exp \left(-3 C_{D} \sqrt{a d}\right) .
$$

For $C_{D}=1,(62)$ has a maximum of about 0.15 at $a d \approx 0.03$. In a random array, interactions will be less since cylinders are staggered. With this justification to neglect the cross-terms, $D_{s}$ is determined by summing the total contribution of all autocorrelation terms $\left\langle u_{i}^{\prime}\left(x_{o}\right) u_{i}^{\prime}(x)\right\rangle$ to dispersion in the unit cell. This is equivalent to integrating $\left\langle u_{1}^{\prime}\left(x_{o}\right) u_{1}^{\prime}(x)\right\rangle$ over all space. Thus, extending the limits of integration in (59) to $\pm \infty$ and using the disturbance of only the fixed cylinder (35) yields

$$
D_{s}=U_{o} d \frac{\sqrt{C_{D}^{3} R e_{t} S c_{t} /\left(S c_{t}+1\right)}}{8 \sqrt{\pi}} \exp \left[-2 x_{o}^{*} C_{D} a d /\left(S c_{t}+1\right)\right] \Gamma\left(\frac{1}{2}, 2 x_{o}^{*} C_{D} a d\right) .
$$

This expression can be written in terms of the variance of the Eulerian velocity field using $(51 a)$ :

$$
D_{s}=2 \sigma_{w}^{* 2} s^{*} \sqrt{\frac{S c_{t}}{S c_{t}+1}} U_{o} d+O(\sqrt{a d}) .
$$

where $\sigma_{w}^{* 2}=\sigma_{w}^{2} / U_{o}^{2}$. Higher-order correction terms are smaller than the error associated with the assumptions made to obtain (63), and are not computed. Note that, in light of (52), $\sigma_{w}^{* 2}$ can be accurately replaced by the total variance, $\sigma_{u}^{* 2}$, in (64).

The above analysis can be carried out for the compensating flows by substituting $u_{g}^{\prime}$, (41), into (59) rather than $u_{w}^{\prime}$. Doing so, we find that the gap contribution to dispersion is

$$
D_{g}=U_{o} d \frac{C_{D} a d}{4(1-a d)}=2 \sigma_{g}^{* 2} U_{o} d
$$

where $\sigma_{g}^{* 2}=\sigma_{g}^{2} / U_{o}^{2}$. From (64) and (65), $D_{g}$ is $O(a d)$ compared with $D_{s}$. This is reasonable given that $\sigma_{g}^{2} \ll \sigma_{w}^{2}$ for small $a d$. However, since $D_{g}$ is proportional to $a d$, it increases as density increases, while $D_{s}$ remains approximately constant. It is thus relevant to compare $D_{g}$ with the contribution from vortex trapping, $D_{v}$, which also increases in proportion to $a d$. In $\S 6$, it is demonstrated that $D_{g} \ll D_{v}$ in nearly all cases of interest.

From (64), $D_{s}$ is proportional to $\sigma_{w}^{* 2}$ (the intensity of spatial velocity fluctuations) and also to the correlation length scale, $s^{*}=1 / \sqrt{a d}$, arising from the $(1 / \sqrt{x}) \exp \left(-C_{D} a x\right)$ wake attenuation. The exponential decay of the defect, (35), ensures that the correlation length scale is finite, and thus, $D_{s}$ exists, i.e. Fickian dispersion. The scaling for $D_{s}$ in (64) is similar to that obtained by Dagan (1987) for dispersion in porous media, for which mechanical dispersion is given by $K=U I \sigma_{Y}^{2}$, where $I$ is the correlation length scale and $\sigma_{Y}^{2}$ is the variance of the log-normally distributed hydraulic conductivity field. Moreover, when non-dimensionalized by the molecular diffusivity, both the wake and gap contributions to dispersion scale with 
the Péclet number,

$$
\begin{gathered}
D_{s} / D_{m} \approx 2 \sigma_{w}^{* 2} s^{*} \sqrt{\frac{S c_{t}}{S c_{t}+1}} P e, \\
D_{g} / D_{m}=\frac{C_{D} a d}{4(1-a d)} P e
\end{gathered}
$$

like the $P e$ scaling found by Koch \& Brady (1985) for mechanical dispersion in Stokes flow through a sphere array.

From (64), $D_{s}$ has only a weak inverse dependence on Schmidt number, which supports our hypothesis that the details of particle transport within the wake are secondary to the macroscopic wake structure. Specifically, consider two extreme scenarios: (i) $S c_{t} \rightarrow \infty$ : particles remain on the time-averaged streamlines without deviation, yielding perfectly correlated trajectories through the wake; (ii) $S c_{t}=1$ : rapid diffusive mixing in the wake makes particle trajectories highly uncorrelated within the wake. From (64), the difference between these two scenarios amounts to a factor of only $\sqrt{2}$ in $D_{s}$. It is reasonable to expect that the real particle trajectories fall between these two scenarios; particles will move transversely through the wake, but in coherent motions dictated by the shedding vortices. The relative independence from the details of correlated motion within a wake justifies our approximation of the Lagrangian correlation by advection through the Eulerian velocity disturbance. Thus, we conclude that (64) accurately reflects the leading-order scaling and provides a good approximation of secondary-wake dispersion.

Furthermore, observe from (64) that $D_{s}$ is only weakly dependent on $a d$. From (52), $\sigma_{w}^{* 2} \propto \sqrt{a d}$, but the length scale $s^{*}=1 / \sqrt{a d}$, such that the two factors cancel. The dependence on ad comes only from $\Gamma$, which is weakly $a d$-dependent. This is an important result, since it qualitatively separates secondary-wake dispersion from vortex-trapping dispersion, which, to leading order and for small $a d$, increases in proportion to ad according to (19). So, we anticipate that as $a d \rightarrow 0, D_{s}$ will be dominant, but as ad increases, vortex-trapping dispersion may grow sufficiently to become the dominant mechanism of dispersion. These trends are born out in experiments discussed in $\S 5$.

Koch \& Brady (1985) find a similar separation between mechanical and boundarylayer dispersion in arrays of spherical elements. Boundary-layer dispersion grows in proportion to the array density, whereas mechanical dispersion is independent of density owing to the effect of array drag in opposing the velocity disturbance associated with each element, known as Brinkman screening (Koch, Hill \& Sangani 1998). This similarity suggests a generalization for dispersion through distributed objects. Trapping dispersion mechanisms associated with the velocity field local to individual objects grow in proportion to the density of individual objects, whereas dispersion associated with the velocity field downstream of individual elements is more weakly dependent on the density.

Finally, secondary-wake dispersion approaches a Fickian diffusion process asymptotically in accordance with the central limit theorem. The Fickian regime is reached after each particle has sampled several different wakes, which will occur when the tracer has been transported for a time $t \gg s / U_{o}$, or

$$
t \gg \frac{d}{U_{o} \sqrt{a d}} .
$$




\subsection{Effects of high packing density on dispersion}

As discussed in $\S 2.1$, for packing densities of approximately 0.1 and higher, significant wake interactions may result and impact the dispersion processes. At high packing density, the array will not behave as a collection of independent elements, but rather as clusters of elements with coalesced wakes acting as large trapping zones, and with streams threading between them. In general, the clustered trapping regions, consisting of the elements and the fluid space between them, will have a proportionally larger stagnant and recirculation zone than the same number of independent elements. As the size of the trapping zones increases, the residence times within these zones will likewise increase. Both trends will tend to enhance trapping dispersion. The theoretical framework presented here for vortex trapping will apply, but the residence time, $\bar{\tau}$, and the relative volume of traps, $\epsilon$, must be appropriately increased, with a dependence that must be determined empirically. At this high density, trapping dispersion will probably make the dominant contribution to longitudinal dispersion, overshadowing secondary-wake dispersion.

\section{Experimental results}

Tracer dispersion was measured in a random array of circular cylinders with diameter $0.64 \mathrm{~cm}$. The cylinder configuration was generated randomly in MATLAB, the location of each cylinder chosen from a uniform p.d.f., with the stipulation that no portion of any two cylinders could overlap. The cylinders were held in Plexiglas base plates placed along the bed of a glass-walled recirculating flume, with water depth $h=15 \mathrm{~cm}$ and width $B=38 \mathrm{~cm}$. The cylinders extended through the free surface. Three array densities were considered: $a d=0.013, a d=0.025$ and $a d=0.082$, which are all within the range of validity for the theories presented.

The flow was controlled by a Weinman 3G-181 recirculating pump and a diaphragm valve to achieve Reynolds numbers, $R e=U_{o} d / v$, ranging between 65 and 650 . To create smooth inlet conditions, energy was dissipated with two mats of rubberized coconut fibre and flow curvature was removed by a $0.45 \mathrm{~m}$ honeycomb flow straightener. The cylinder array began $3 \mathrm{~m}$ from the inlet.

For each density, the spatially and temporally varying velocity field was characterized using a SonTek MicroADV acoustic Doppler velocimeter sampling at $50 \mathrm{~Hz}$. Transverse profiles were made at several streamwise locations and depth profiles were made at several horizontal locations. These measurements are summarized in figure 3. The horizontal velocity field (figure $3 a$ ) exhibits strong variability owing to the random distribution of cylinders. A boundary layer near each wall, which is very small compared with the channel width, is the only exception to the spatial randomness. The depth variation at a single horizontal coordinate (figure $3 b$ ) exhibits much less variability, and is approximately uniform except for a boundary layer near the bed which is small compared with the total depth. As the boundary layers are confined to very thin regions, and depth variation is small, the treatment of the velocity field as a random function of only the horizontal coordinate is justified.

Histograms showing the scatter in the spatial velocity measurements are given in figure 4 and the dependence of the spatial velocity variance on Reynolds number and cylinder density are shown in figure 5. As predicted by the theory, (51a) and (52), the variance of $u(x, y)$ increases with $a d$, and the data, though not extensive, suggest the leading-order scaling $\sigma_{u^{\prime}}^{2} \sim \sqrt{a d}$ is appropriate, as shown by figure 5 . In addition, the variance is greater for low $R e$, which, given the well-established behaviour for an isolated cylinder of decreasing drag coefficient with increasing Reynolds-number, is 

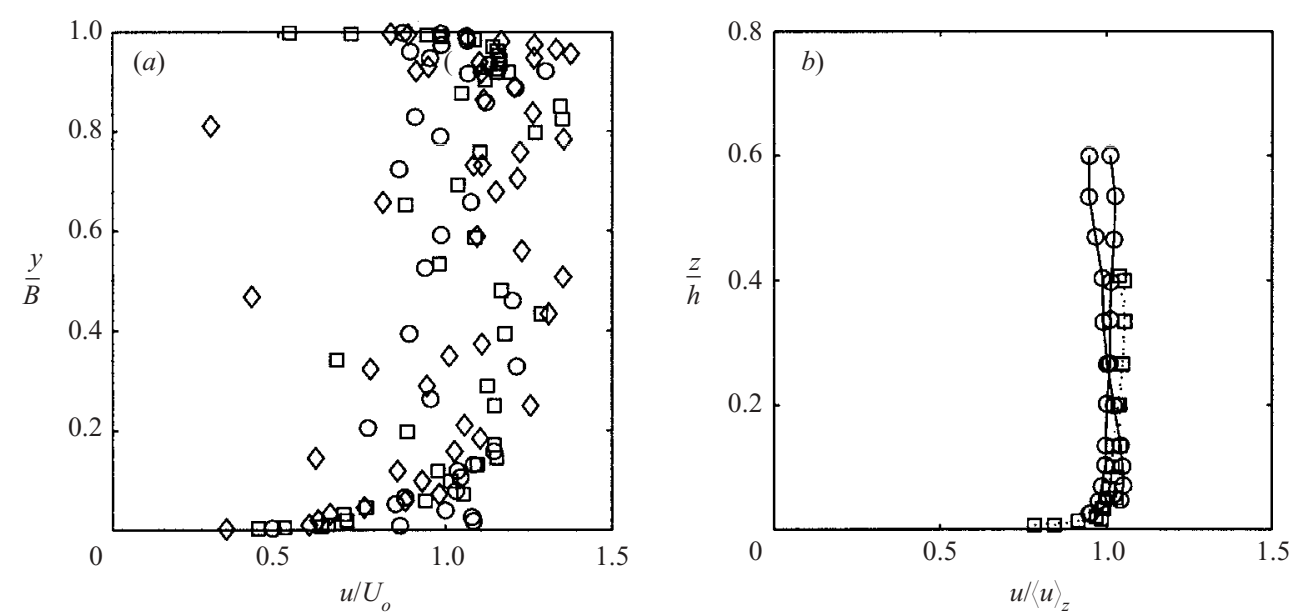

FIGURE 3. Spatial variability in the velocity field. (a) Transverse profiles of streamwise velocity for $R e=660$ and $a d=0.013$, measured at 3 different streamwise locations, spaced approximately $115 d$ apart, and denoted by circles, squares and diamonds. The locations of the profiles were chosen at random from within the array, while maintaining the given spacing between any two profiles. $(b)$ Depth profiles at various horizontal coordinates for $R e \approx 600$ and $a d=0.013$ (squares, dotted line), and $a d=0.082$ (circles, solid line). Velocity is normalized by the depth average, $\langle u\rangle_{z}$. The uncertainty in all velocity measurements is within the size of the marker. Note that variability is much more pronounced with respect to the horizontal coordinate than the vertical. Thin boundary layers are observed near the bed (below about $0.03 h$ ) in the vertical profiles, and near the walls (inside about $0.03 B$ ) in the lateral profiles. These observations support a model of the velocity field as a random function of horizontal coordinate, $u(x, y)$.

consistent with the $\sigma_{u}^{2} \propto C_{D}^{3 / 2}$ relationship predicted by (51a). The Reynolds-number dependence of $\sigma_{u^{\prime}}^{2}$ is shown in figure 6 .

The velocity variance measured here for sparse arrays $(\alpha<0.1)$ can be contrasted with those of Hill \& Koch (2002) for flows in the moderate-Reynolds-number range, but in a more dense periodic array $(\alpha=0.2)$. Their results for the total variance, which include temporal fluctuations and fluctuations within the primary wakes (not included in our analysis) show that the variance increases with Reynolds number as the flow becomes increasingly chaotic. The results further show the effect of close cylinder spacing, made even more pronounced by the periodic nature of their array, which leads to primary-wake interactions, as discussed in $\S 2.1$. Such effects are not present in our random array at lower density and may explain why we observe different behaviour to Hill \& Koch.

To characterize the decay of the wake velocity deficit, we measured the mean correlation, $r=\left\langle\left(u_{1}-U_{o}\right)\left(u_{2}-U_{o}\right)\right\rangle / \sigma_{u^{\prime}}^{2}$, between two successive profiles, $u_{1}(y)$ and $u_{2}(y)$, separated by distances of $(79-118) d$, or $(1-4) / a$ depending on $a d$. The three profiles shown in figure 3(a) are typical of these separated profiles. For all $a d$, we found $r<0.10$, consistent with the exponential decay of velocity fluctuations, $1 / \sqrt{x^{*}} \exp \left(-C_{D} a d x^{*}\right)$, from $(35)$ which predicts $r<0.02$ for these spacings and densities.

Tracer experiments were conducted by injecting small pulses of Rhodamine WT dye into the flume at mid-depth and mid-width at a distance $0.63 \mathrm{~m}(\approx 100 d)$ into the array, and this position is denoted $X=0$. Based on results for the longitudinal decay in correlation between cross-sectional velocity profiles just discussed, it was 

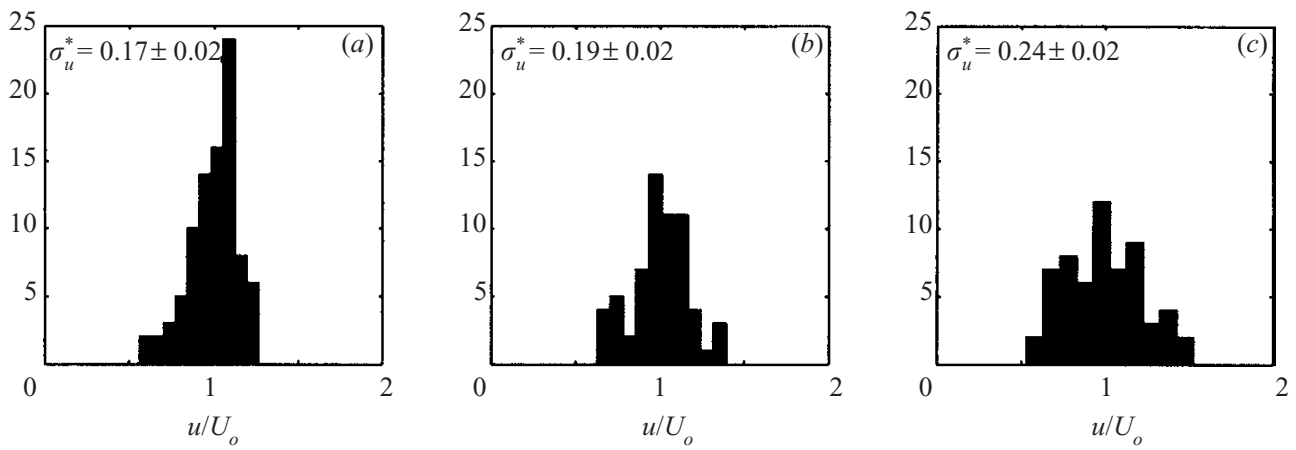

FIGURE 4. Histograms of spatial velocity measurements for $R e \approx 650$ and $(a)$ ad $=0.013$, (b) 0.025 , and $(c)$ 0.082. Data are taken from two lateral velocity profiles for each case. The standard deviation, $\sigma_{u}^{*}=\sigma_{u} / U_{o}$, is shown in the plots.

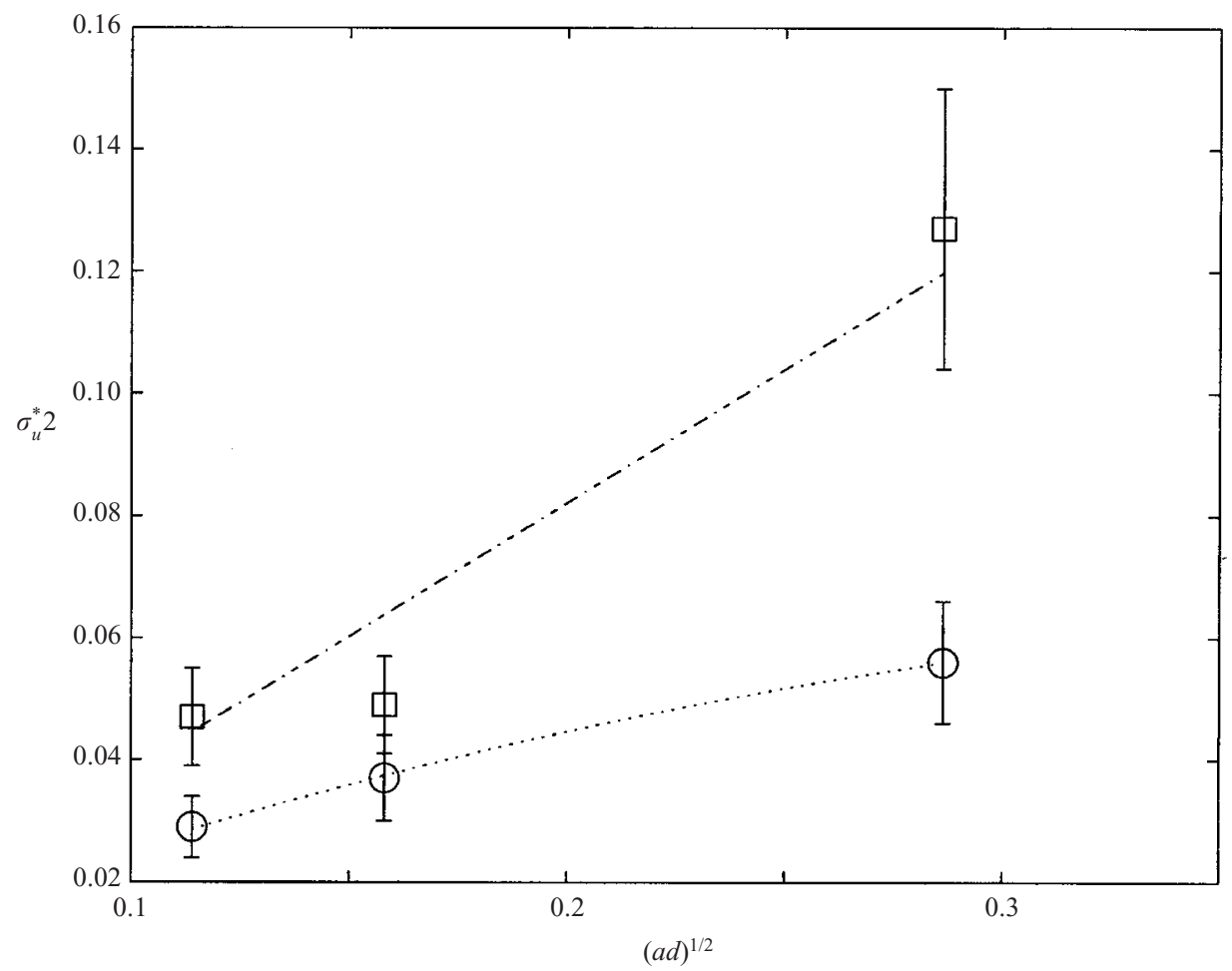

FIGURE 5. Relationship between spatial velocity variance, $\sigma_{u}^{* 2}=\sigma_{u}^{2} / U_{o}^{2}$, and ad for $R e \approx 650$ (circles) and $R e \approx 65$ (squares). Theory, (51a) and (53), predicts the relationship $\sigma_{u}^{* 2}=(1 / 16) \sqrt{C_{D} R e_{t}} \sqrt{a d}-(1 / 4 \sqrt{2 \pi}) C_{D}^{2} \sqrt{\operatorname{Re}_{t} x_{o}^{*}} a d$, i.e. second order in $\sqrt{a d}$. Least-squares second-order polynomial fits were performed on the data for each Reynolds number with $C_{D}$ and $x_{o}$ the adjustable parameters and using $R e_{t}=35$, obtained from data in Kovasznay (1949) and Zavistoski (1994). The best fits, shown by the dotted line $(R e \approx 650)$ and the broken line $(R e \approx 65)$ yielded for $R e \approx 650$ values of $C_{D}=0.85$ and $x_{o}^{*}=0.58$, both well within the expected range. For $R e \approx 65$, the second-order fit yielded a positive coefficient for the second term, which is unphysical, so a linear fit was performed to yield $C_{D}=1.1$, also quite reasonable. The decrease in drag coefficient with increasing Reynolds number, as suggested by the fits, is consistent with the drag dependence for an isolated cylinder (Schlichting 1987). The consistent increase in $\sigma_{u}^{* 2}$ with $a d$ and the reduction for higher $R e$ are both consistent with theory as given by $(51 a)$. 


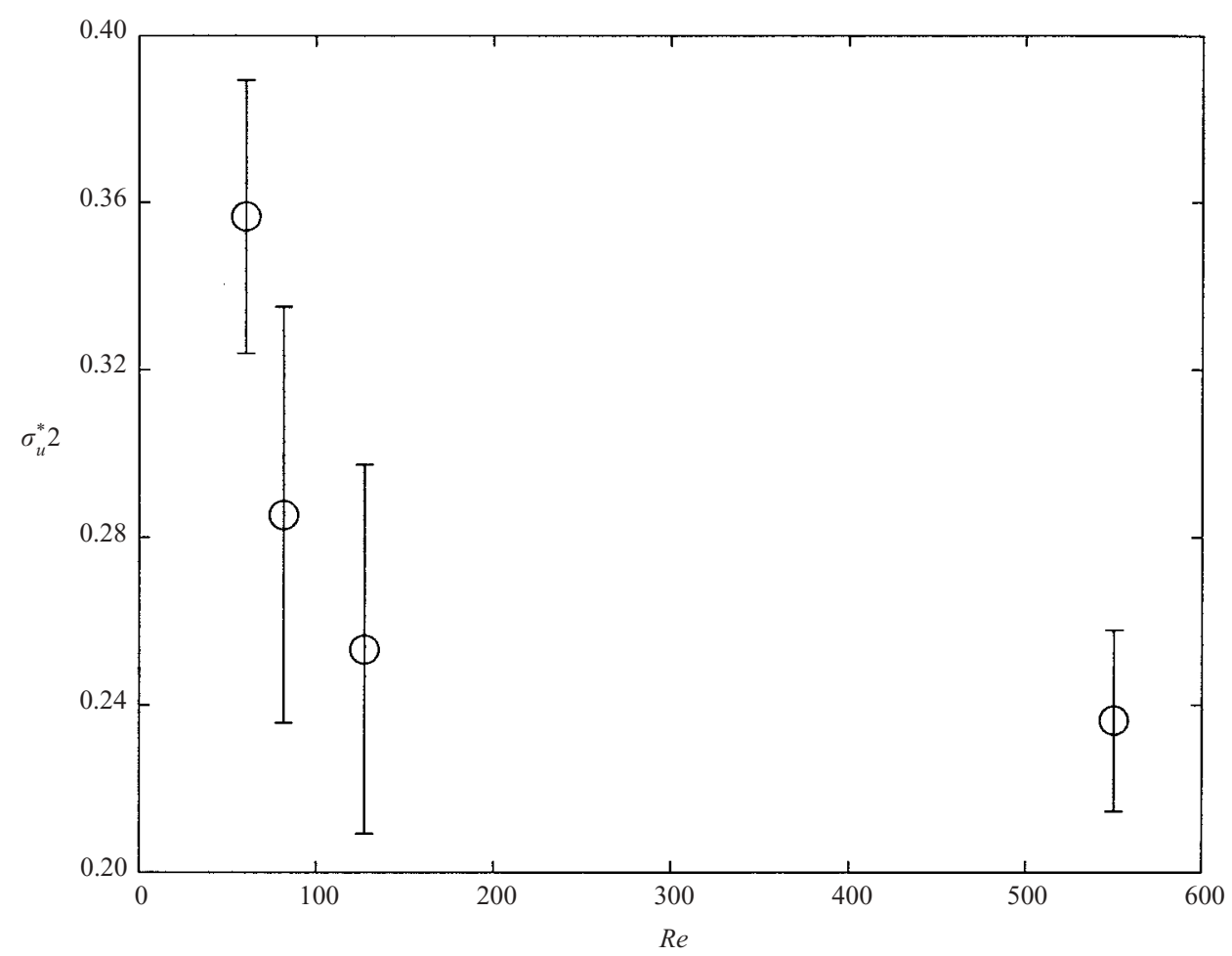

FIGURE 6. Dependence on $R e$ of the standard deviation, $\sigma_{u}^{*}=\sigma_{u} / U_{o}$, of the velocity field for $a d=0.082$. Uncertainties due to the finite number of measurements (samples of the underlying velocity distribution) are shown by vertical bars. $\sigma_{u}$ is inversely related to $R e$, consistent with the well-established $C_{D}-R e$ relationship for an isolated cylinder in this $R e$ range. This supports the theoretical relationship $\sigma_{u}^{2} \propto \sqrt{C_{D}^{3}}$ predicted by $(51 a)$.

concluded that the flow at $X=0$ was representative of flow conditions throughout the array, showing no signature from the flow upstream of the array. Time records of dye concentration were measured using a Chelsea Instruments Aquatracka III fluorometer (sampling rate $7.5 \mathrm{~Hz}$ ) placed at a fixed downstream location which ranged from $X=0.5 \mathrm{~m}$ to $X=3.5 \mathrm{~m}$ over separate tracer releases. At least 10 realizations were performed for each experimental scenario. Concentration records were normalized according to

$$
C^{*}(t)=\frac{C(t)}{\int_{-\infty}^{\infty} C(t) \mathrm{d} t},
$$

where $C^{*}(t)$ is the density function for tracer passage time. This normalization removes variations in $C(t)$ across realizations caused by small differences in the mass of dye released and also eliminates the effects of transverse and vertical diffusion and drifting of the tracer cloud. At each longitudinal position $X$ and for each realization, denoted by $i$, the mean arrival time, mean velocity, and temporal variance of the tracer cloud are given by

$$
\overline{t_{i}}=\int_{-\infty}^{\infty} t C_{i}^{*}(t) \mathrm{d} t
$$



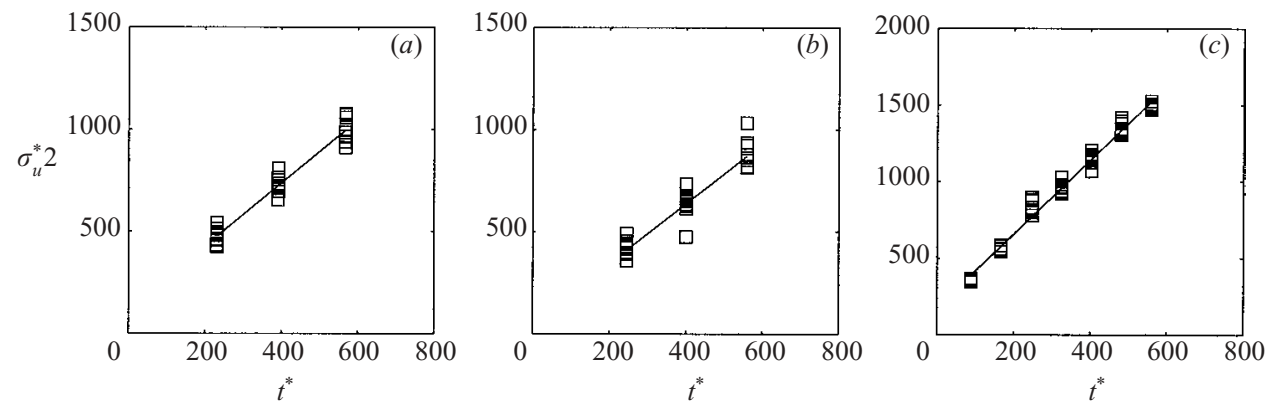

FigURE 7. Growth of tracer variance with time for $R e \approx 600$ and $(a)$ ad $=0.013,(b) 0.025$ and $(c)$ 0.082. The normalized spatial variance, $\sigma_{x}^{* 2}=\sigma_{x, i}^{2} / d^{2}$, for each individual realization, is plotted on the $y$-axis, while the normalized time, $\bar{t}_{i}{ }^{*}=\overline{t_{i}}\left(U_{i} / d\right)$ is plotted on the $x$-axis. For a Fickian process, the variance grows linearly with time. The effective dispersion constant is given by the linear best fit, i.e. $D / U_{o} d=\frac{1}{2}\left(\mathrm{~d} \sigma_{x}^{* 2} / \mathrm{d} \overline{t^{*}}\right)$.

$$
\begin{gathered}
U_{i}=\frac{X}{\bar{t}_{i}}, \\
\sigma_{t, i}^{2}=\int_{-\infty}^{\infty} t^{2} C_{i}^{*}(t),
\end{gathered}
$$

from which the ensemble averages $\langle\bar{t}\rangle,\langle U\rangle$ and $\left\langle\sigma_{t}^{2}\right\rangle$ can be computed. The spatial variance is calculated by the kinematic relationship, $\sigma_{x, i}^{2}=U^{2} \sigma_{t, i}^{2}$. This conversion is valid for large Péclet number based on longitudinal dispersion, $P>100$ (Levenspiel \& Smith 1956), which is satisfied in these experiments.

For Fickian diffusion, tracer variance grows linearly in time with the dispersion constant determined from the slope $D=\frac{1}{2}\left(\mathrm{~d} \sigma_{x}^{2} / \mathrm{d} t\right)$. Introducing the non-dimensional variance and time

$$
\begin{aligned}
\sigma_{x, i}^{* 2} & =\sigma_{x, i}^{2} / d^{2}, \\
t^{*} & =t U_{i} / d,
\end{aligned}
$$

the non-dimensional dispersion constant is

$$
D^{*}=\frac{D}{U_{o} d}=\frac{1}{2} \frac{\mathrm{d} \sigma_{x}^{* 2}}{\mathrm{~d} t^{*}} .
$$

Results for all $R e-a d$ scenarios show linear growth of variance with time, with correlation coefficients, $r^{2}$, typically well above 0.9 , indicating a highly significant correlation (Taylor 1997). Typical plots are shown in figure 7.

That the tracer variance grows linearly with time (figure 7) suggests that the second moment of the tracer distribution evolves by Fickian dispersion. We can confirm whether the experimental array is indeed long enough for the processes expected to be contributing to dispersion to have reached the Fickian limit. From (68), multiplying by the mean velocity to convert the time scale to a distance, the asymptotic distance necessary for secondary-wake dispersion to become Fickian is much greater than $O(d / \sqrt{a d})$ or much greater than $\approx 2 \mathrm{~cm}$ and $\approx 6 \mathrm{~cm}$, for the density scenarios $a d=0.082$ and $a d=0.013$, respectively. Thus, the observation of Fickian dispersion within the $350 \mathrm{~cm}$ experimental array is consistent with the prediction. Moreover, from (21), neglecting scale constants and converting the time scale to a distance, the vortex-trapping theory, (21), predicts that the tracer must advect 


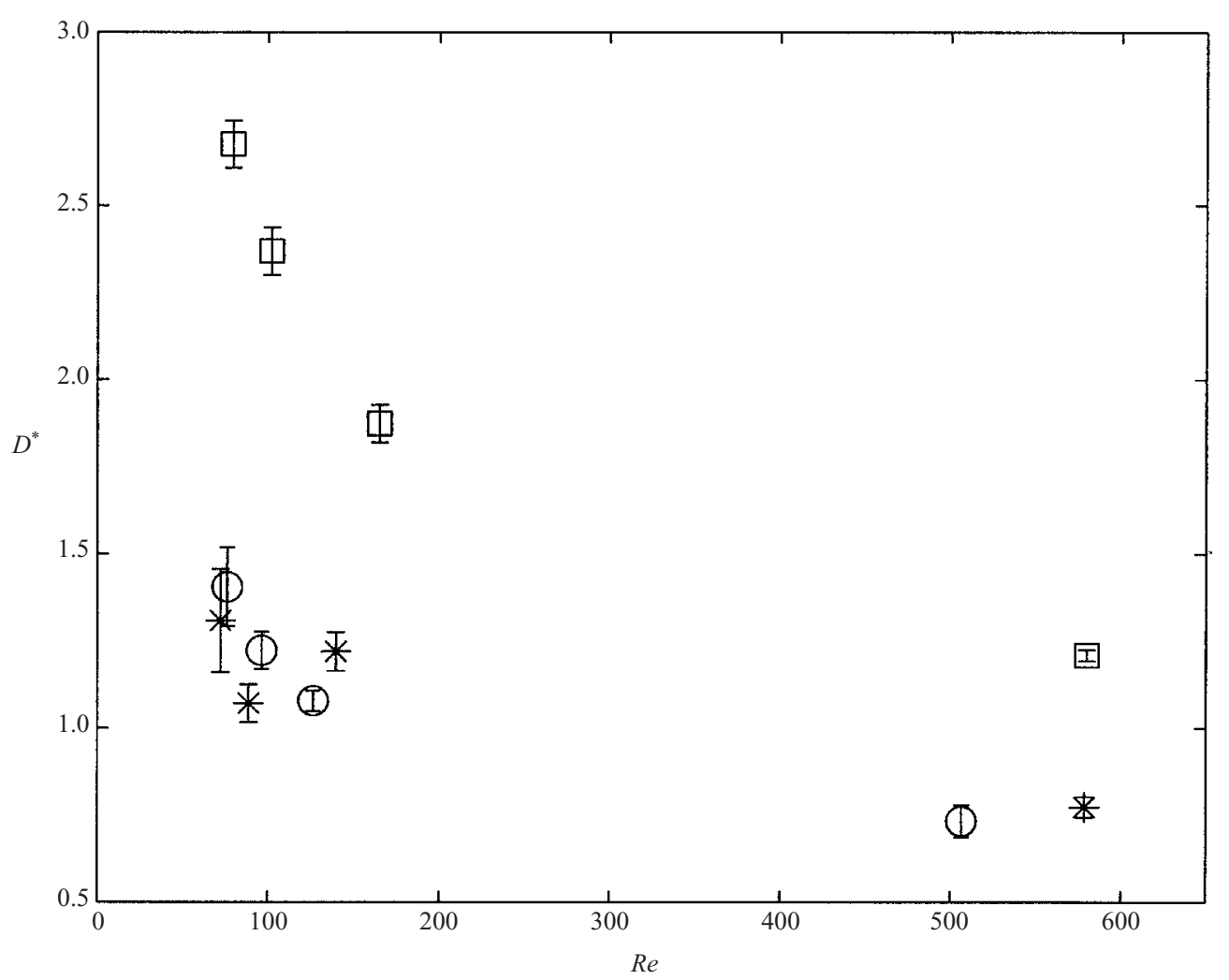

Figure 8. Dependence of total dispersion, $D^{*}=D / U_{o} d$, on $\operatorname{Re}$ for $a d=0.013(*), a d=0.025$ (circles), $a d=0.082$ (squares). Vertical bars give uncertainty in $D^{*}$ obtained from linear regression analysis of $\sigma_{x}^{* 2} v s . t^{*}$ data. The inverse relationship between $R e$ and $D^{*}$ observed for all ad scenarios is consistent with the dependence of both $D_{s}$ and $D_{v}$ on the changing wake structure. The primary wake size and residence time, as well as the drag coefficient, all decrease with increasing $R e$. For all $R e, D^{*}$ is comparable at $a d=0.013$ and $a d=0.025$, but increases sharply at $a d=0.082$. This suggests that at the lower $a d$ secondary-wake dispersion dominates, but is surpassed by vortex-trapping dispersion at the highest $a d$.

a distance at least $O(1 / a)$ before the distribution is Gaussian. This corresponds to distances in the range $O(10)$ and $O(100) \mathrm{cm}$ for the density scenarios $a d=$ 0.082 and $a d=0.013$, respectively. Thus, the observation of Fickian dispersion within the experimental array is also consistent with the vortex-trapping prediction. However, from (29), the length scale for boundary-layer dispersion to be significant is $O\left(\left(c_{f} R e\right)^{-1 / 3} P e^{2 / 3} / a\right)$. For our experiments, $P e=O\left(10^{5}\right), R e=O(100), c_{f} \approx 5.93 /$ $\sqrt{R e}$, and thus the asymptotic time scale must be at least $\approx 5000 \mathrm{~cm}$ and $\approx 30000 \mathrm{~cm}$ for the density scenarios $a d=0.082$ and $a d=0.013$, respectively, which is much longer than the $350 \mathrm{~cm}$ experimental array. Thus, we do not think that boundary-layer dispersion due to the cylinder surfaces played any role in the dispersion of the tracer cloud in the experiments.

In figure 8, the measured dispersion constants are plotted as a function of $R e$ for each $a d$ scenario. There are two clear trends: (i) for all $a d, D^{*}$ decreases with increasing Reynolds number, and (ii) for all $R e, D^{*}$ increases sharply for the highest ad after changing very little between the low and middle $a d$. The first dependence, $D^{*}=f(R e)$, is consistent with changes in both the primary and secondary wake 
structure with Reynolds number. The drop in $D^{*}$ is most dramatic between $R e=65$ and 200, and corresponds well to similar changes in the size of the vortex-formation zone (primary wake) across this range observed by Gerrard (1978). Gerrard also observed that near $R e=100$, the residence time of the primary wake drops sharply (Gerrard 1978) as convection of vorticity begins to dominate diffusion. According to the therory developed in $\S 3$, this decrease in the size of the primary wake and its residence time would contribute to a decrease in trapping dispersion with increasing $R e$, consistent with theory. Furthermore, it is well-established that the drag coefficient drops by a factor of nearly two between $R e=65$ and 200. With this drop in $C_{D}$, (51a) predicts a decline in $\sigma_{u}^{2}$, which is supported by the observations in figures 5 and 6 , and thus a decline in secondary-wake dispersion, as given by (64).

The dependence of $D^{*}$ on ad observed in figure 8 is also consistent with theory. Recall that vortex-trapping dispersion increases in proportion to $a d$, whereas secondary-wake dispersion is only weakly dependent on $a d$. That $D^{*}$ does not increase between $a d=0.013$ and 0.025 suggests that secondary-wake dispersion dominates the total dispersion in this range. However, when $a d$ increases to $0.082, D^{*}$ increases sharply, suggesting that between $a d=0.025$ and 0.082 , the contribution of trapping dispersion surpasses that of secondary-wake dispersion and begins to control the dependence of $D^{*}=f(a d)$.

\subsection{Cylinder-scale mixing}

The theory and results presented thus far have been obtained by averaging over an area much larger than the cylinder spacing, and thus do not describe mixing on smaller scales. Now we consider the transport at scales smaller than $s$, particularly to highlight a transition in behaviour occurring near $R e \approx 200$. Figure 9 depicts concentration time series, $C^{*}(t)$, measured at different Reynolds numbers. Significant fluctuations in the time series are evident for $R e=65$ (figure $9 a$ ), but are absent from the profiles at $R e=650$ (figure $9 b$ ). The strength of the fluctuations are characterized by $C_{r m s}^{*}=\left\langle C_{i}^{*}(t)-\overline{C^{*}(t)}\right\rangle /\left(\max \overline{C^{*}}(t)\right)$ where the overbar denotes an average over all realizations for each time and the angle brackets an average over the time series. A dramatic decrease in fluctuation strength occurs between $R e=65$ and $R e=650$, with $C_{r m s}^{*}$ dropping from 0.07 to 0.01 .

Insight into these fluctuations can be gained from the spectral density plots of both concentration and transverse velocity, as shown in figure 10. Peaks in the velocity spectra corresponding to vortex-shedding frequencies, with Strouhal numbers of $S t=f d / U_{o} \approx 0.16$, are evident in both the $R e=650(10 a)$ and $R e=65(10 b)$ scenarios. The peaks in the concentration spectra, however, occur at frequencies lower than those of vortex shedding. For $R e=650$, the peak frequency corresponds to the time scale of the full tracer cloud form advecting past the measurement point. The absence of higher frequencies, i.e. small-scale heterogeneity, is consistent with the smoothly varying tracer distribution apparent in figure $9(b)$. For low $R e$, however, a spectral peak occurs between $0.07 \mathrm{~Hz} \lesssim f \lesssim 0.1 \mathrm{~Hz}$. This frequency peak corresponds to the convection of spatial heterogeneity of length scale $l \sim U_{o} / f \sim 20 d$ for $a d=0.013$, i.e. it is of the same order as the cylinder spacing, $s \approx 9 d$. That the length scale for spatial fluctuations is the spacing scale, $s$, suggests the following. The fluctuations in concentration represent the passage of distinct unmixed tracer filaments. The filaments are stretched and folded through interaction with individual cylinders and their local velocity gradients, such that the distortions have a length scale comparable to the cylinder spacing. Distinct tracer filaments persist at low Reynolds number because the free-stream diffusion is weak. For higher Reynolds number, 

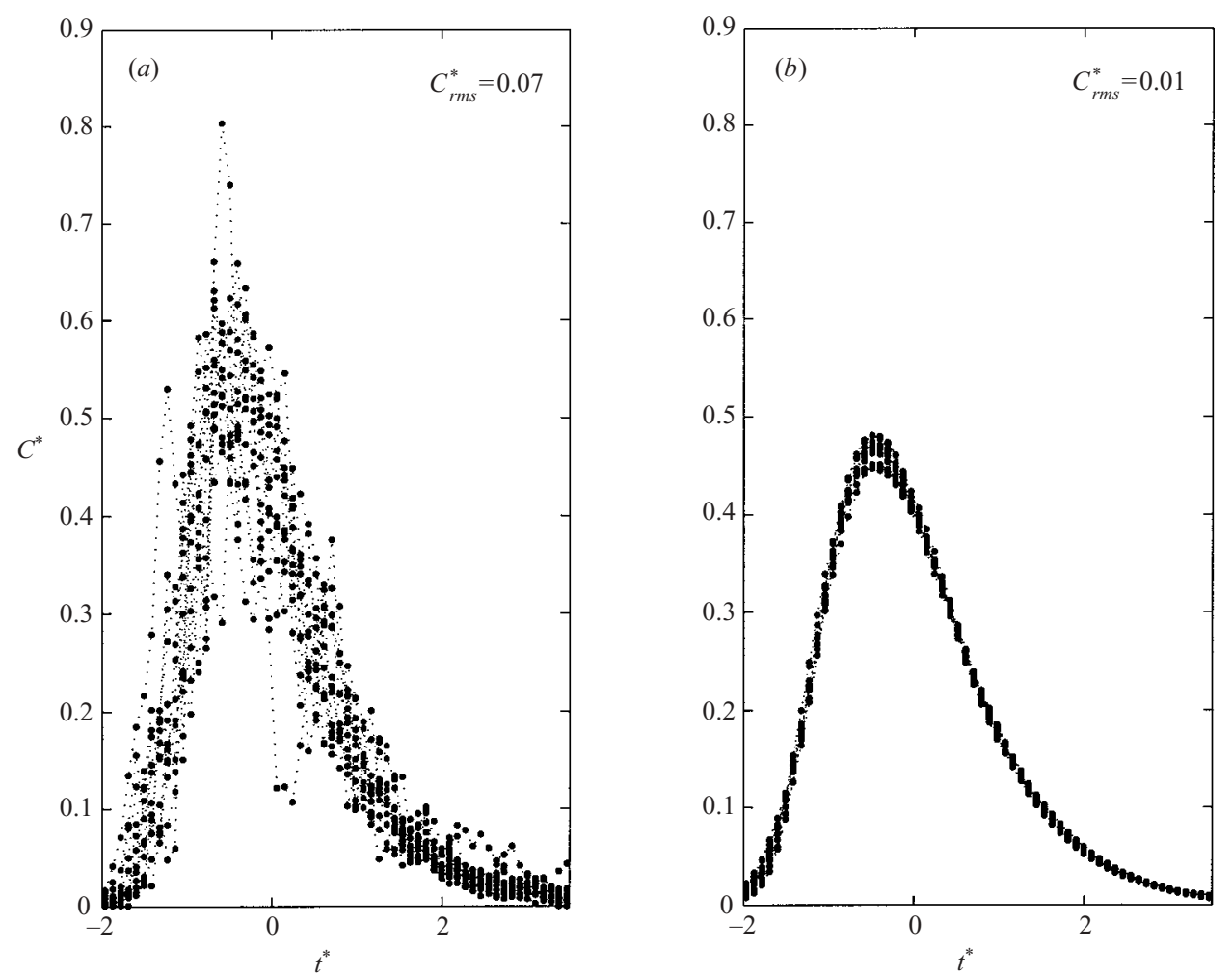

FIGURE 9. Degree of mixing with Reynolds number. Profiles for $(a) R e=65$ and $(b) 650$ and $a d=0.013$ (approximately 10 realizations for each case). Profiles have been rescaled by $t^{*}=\left(t-\overline{t_{i}}\right) / \sigma_{t, i}$ and $C^{*}=C_{i}^{*} \sigma_{t, i}$. Fluctuations are quantified by $C_{r m s}$, the root mean square of the deviations from the ensemble mean; $C_{r m s}^{*}=C_{r m s} / C_{\text {peak }}$. The low $\operatorname{Re}$ profile $(a)$ exhibits significant fluctuations that are absent in the high $R e$ profile $(b)$.

free-stream turbulence generated by the element wakes is sufficient to erase the filament signature and smooth the concentration profiles. The onset of enhanced smallscale mixing occurs when the shedding vortices associated with each element become turbulent. This transition occurs near $R e=200$ for an isolated cylinder (Kundu 1990). Consistent with this transition to turbulence, Nepf, Sullivan \& Zavistoski (1997) observed a dramatic increase in transverse diffusion, a proxy for turbulent diffusion, between $R e=110$ and $R e=190$ in a model array identical to that used here. Hill \& Koch (2002) observed that in an unbounded periodic array with $\alpha=0.2$, a transition occurs near $R e \approx 200$ (using our definition of $R e$ ), below which the velocity field is periodic in time, and above which it becomes chaotic. Presumably, this would correspond to the mixing transition, and it occurs in the Reynolds number range consistent with our observations and the others cited here. It is interesting to note that Hill \& Koch found the transition to be shifted to a smaller Reynolds number $(\approx 80)$ for a wall-bounded array. Our observations are more in line with the transition expected for an isolated cylinder, and that observed by Hill \& Koch in the unbounded array, suggesting that the wall effects were not significant in our random, more sparse array. Also interesting is the fact that we observe very little dependence of the Strouhal number with cylinder density or Reynolds number, as all measurements were between $S t=0.16$ and $S t=0.2$ between $65<R e<650$ 

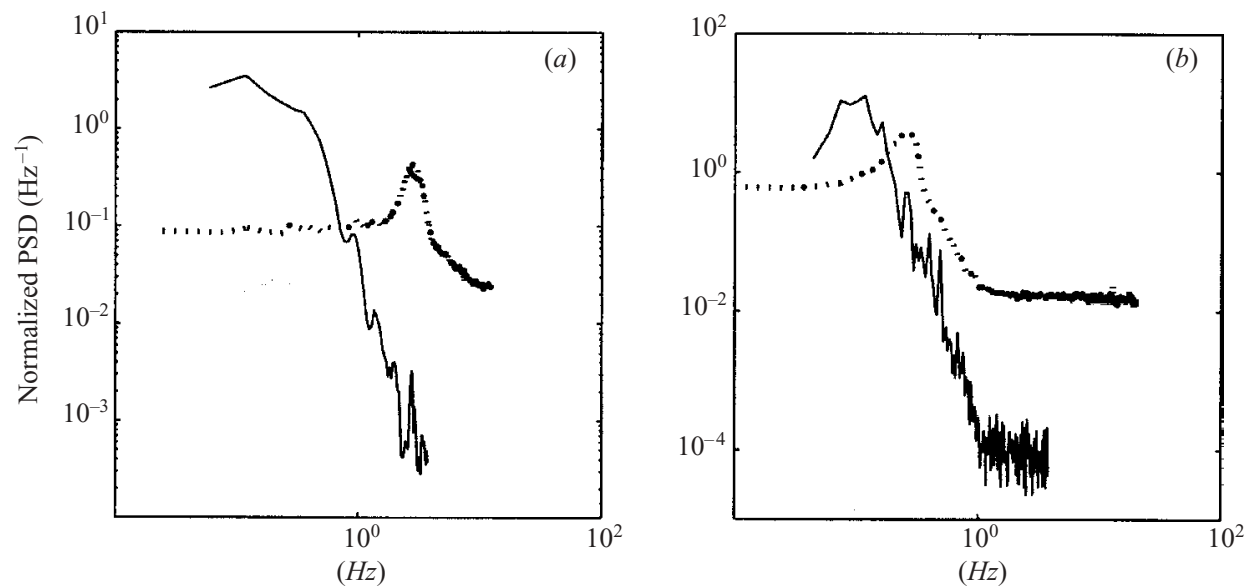

FIGURE 10. Power spectral density plots for temporal fluctuations in both concentration (solid lines) and transverse velocity component (dotted lines) at $a d=0.013$. PSD are normalized such that they are proper density functions, i.e. the integral over frequency space is unity. (a) $R e=650$ corresponds to the concentration profiles in figure $9(b) ;(b) R e=65$ corresponds to figure $9(a)$. Distinct peaks in the velocity spectra $-f \approx 2.5$ for $R e=650 ; f \approx 0.25$ for $R e=65$ - correspond to vortex shedding, with Strouhal number of $S t \approx 0.16$ in each case. The peaks in the concentration spectra are shifted to lower frequency relative to vortex shedding. For low $R e,(b)$, fluctuations are due to convection of spatial disturbances, and the dominant concentration frequencies $-f \approx 0.1$ - correspond to length scales, $l=U_{o} / f$, around $20 d$, which is of the order of the cylinder spacing, $s=d / \sqrt{a d} \approx 9 d$. For high $\operatorname{Re},(a)$, the dominant frequencies correspond to the length scales of the mean tracer distribution, i.e. the cloud is nearly homogeneous on the sub-cloud scale. Finally, note that for both cases, the sampling frequency of the fluorometer can resolve concentration fluctuations covering the full range of the dominant velocity fluctuations.

and $0.013<a d<0.082$, similar to the values for an isolated cylinder (Schlichting 1987). This shows that vortex-shedding dynamics and transition to turbulence for sparse random arrays are less affected by wall effects and cylinder interactions than dense periodic arrays, and, to leading order, indeed behave as a collection of isolated cylinders, as we have assumed.

The profiles in figure 9 allow us to estimate the magnitude of turbulent diffusion. In figure $9(c)$, the spacing-scale fluctuations are absent, implying that the time scale for mixing over the spacing scale is faster than advection to the measurement point. The time scale for diffusion over $s$ is of order $s^{2} / D_{t}$ and for advection is $X / U_{o}$. Then $O\left(s^{2} / D_{t}\right)<X / U_{o}$, and so we have that $D_{t} / U_{o} d \geqslant O(0.1)$. Conversely, in figure $9(a)$ the spacing scale fluctuations persist, so it follows that $D_{t} / U_{o} d \ll 0.1$. These estimates are in agreement with results from Nepf et al. (1997), who found for a comparable cylinder density, $D_{y} / U_{o} d=0.17$ for $R e=588$, and $D_{y} / U_{o} d=0.02-0.06$ for $R e=90-100$.

Based on the previous discussion, it must be noted that when considering only array-scale dispersion, a description of the transport on small scales is elusive. This is an important issue in applications for which local variations in the scalar field are significant, for example, in mass transfer between aquatic plants and the water column, or heat transfer between rods and the surrounding fluid. Many biological processes respond nonlinearly to local concentration, and will be strongly affected by the fluctuations evident in figure 9. 


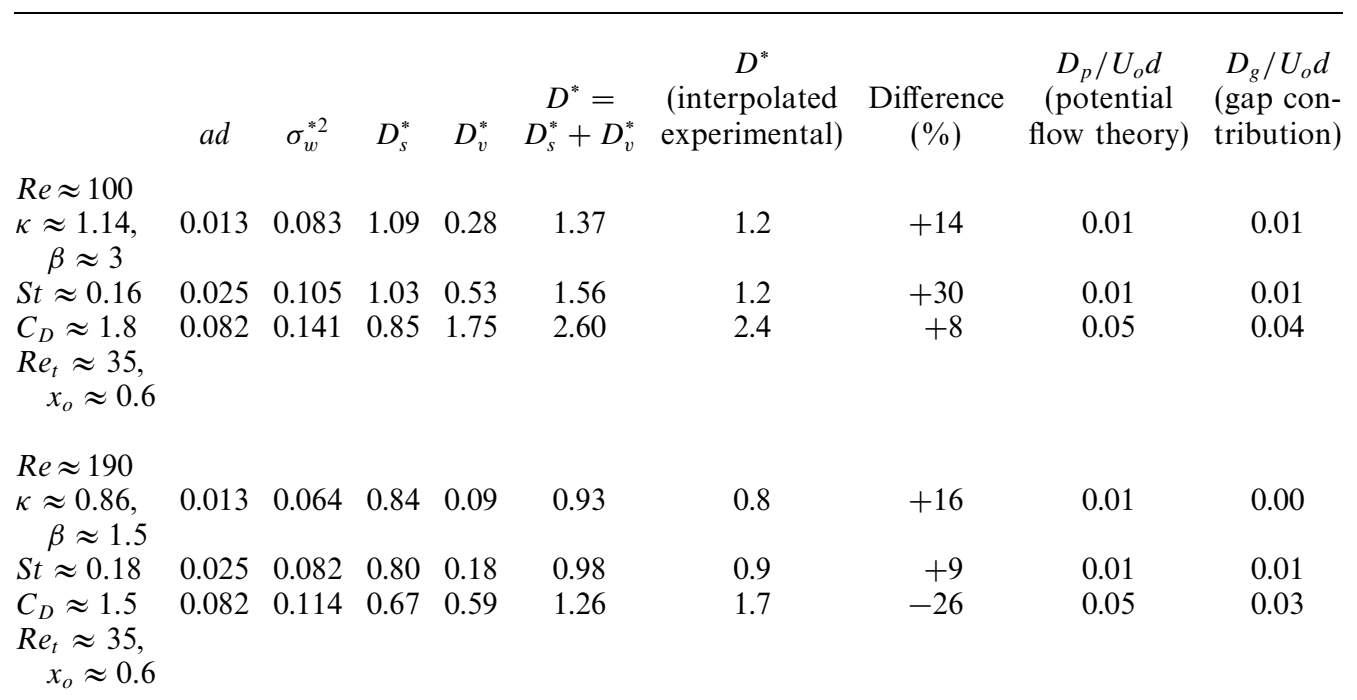

TABLE 1. Comparison of theory with experiment for Reynolds numbers 100 and 190. For each case, $\sigma_{w}^{* 2}$ and $D_{s}^{*}=D_{s} / U_{o} d$ are predicted from (51a) and (65) using the Re-dependent constants $C_{D}$ and $S t$ for an isolated cylinder (Schlichting 1987 figures 1.4 and 2.9); $R e_{t}$ was fitted from experimental wake profiles at $R e=56$ (Kovasznay 1949) and $R e=190,770$ (Zavistoski 1994), which gave $R e_{t} \approx 35 ; x_{o}^{*}=0.6$ is based on the experimental fit shown in figure 5. $D_{v}^{*}=D_{v} / U_{o} d$ is predicted from (19) with the parameters $\kappa$ and $\beta$, taken from Duan \& Wiggins (1997). The theoretical prediction for the total dispersion constant, $D^{*}=D_{s}^{*}+D_{v}^{*}$, is an independent estimate, made without any parameter adjustment. However, since experimental data were not available for the Reynolds numbers analysed by Duan \& Wiggings, $R e=100$ and 190, the experimental values for $D$ were obtained by interpolating the data shown figure 8 . The last two columns show the contributions to total dipersion made by, respectively, the potential flow prediction from Eames \& Bush (1999), (A 1), and the cylinder gap flow, (66). Both are negeligible compared with $D_{v}^{*}$ and $D_{s}^{*}$ across all scenarios

\section{Comparison with theory}

To evaluate further the theory for vortex trapping and secondary-wake dispersion, the predictions for total dispersion based on the theory are directly compared with experimental observations. First, $x_{o}$, and $R e_{t}$ are required to estimate secondary-wake dispersion $D_{s}$. The drag coefficient $C_{D}$ can be estimated based on an isolated cylinder (Schlichting 1987 figure 1.4). Alternatively, reasonable estimates of $C_{D}$ and $x_{o}$ can be made from figure 5 (see caption). We take $R e_{t}=35$, from wake profiles at Reynolds numbers of 56, 190 and 770 taken from data in Kovasznay (1949) and Zavistoski (1994). The effective Schmidt number, $S c_{t}$, is taken to be unity, a good approximation for large Reynolds number (Hinze 1975).

Secondly, to estimate vortex-trapping dispersion, $D_{v}$, the parameters describing the size of the wake-trapping zone, $\kappa$, and the residence time, $\beta$, are necessary. These are non-trivial functions of Reynolds number. Some results are available from Duan \& Wiggins (1997), who used lobe dynamics to compute the size and residence time of the primary wake cavity for $R e=100$ and $R e=190$. From their results, $\kappa$ and $\beta$ can be estimated. The mean residence time is estimated as the time for the fraction $1 / e$ of particles initially residing within the wake to be removed. These parameter values along with others leading to the theoretical predictions of $D_{s}$ and $D_{v}$ are shown in table 1 . Because experimental data were not available for the Reynolds 

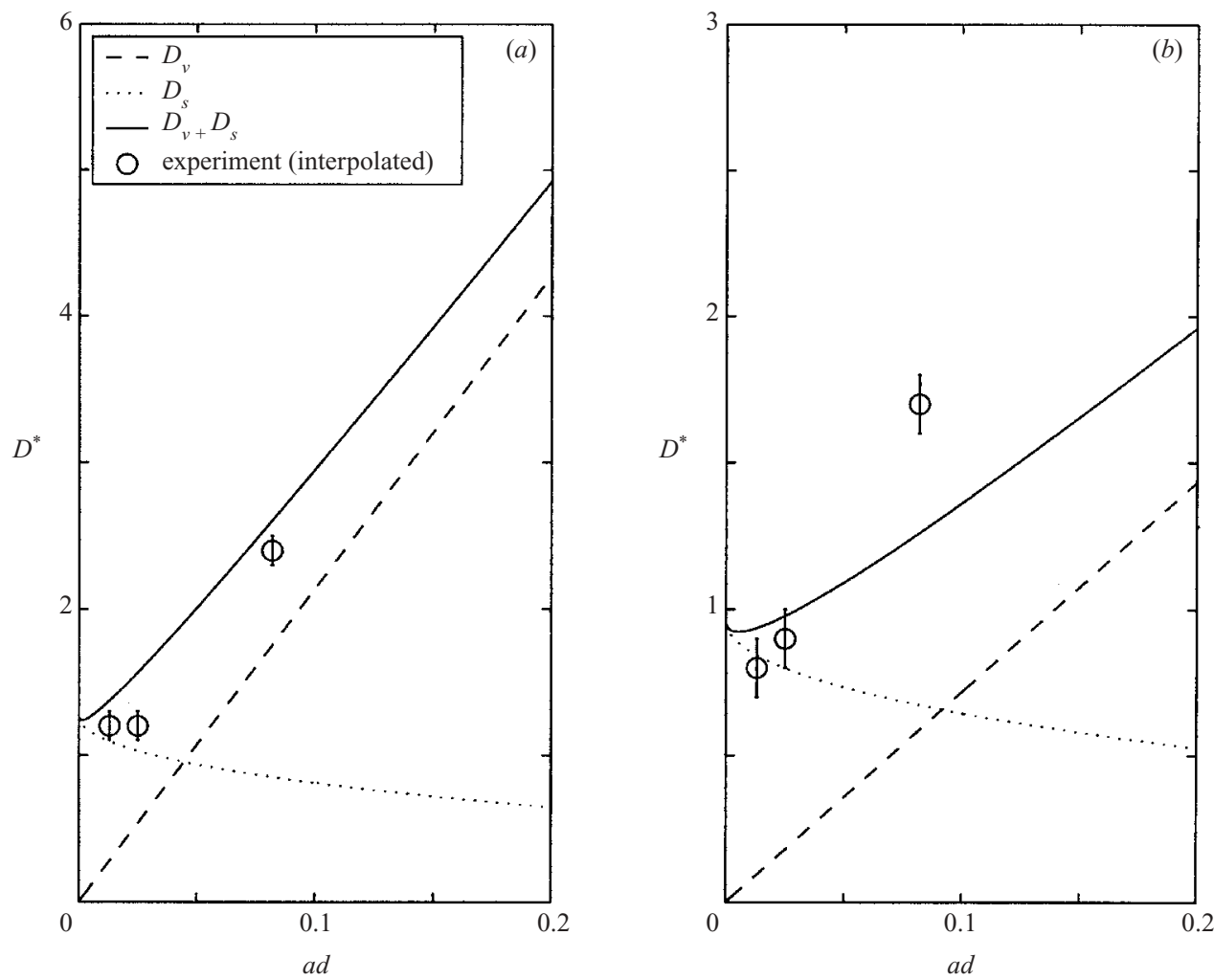

FiguRE 11. Comparison of theory and experiment for $(a) R e=100$ and $(b)$ 190. Theoretical predictions are made for secondary wake dispersion, $D_{s}^{*}=D_{s} / U_{o} d$, and vortex trapping dispersion, $D_{v}^{*}=D_{v} / U_{o} d$, using parameters described in table 1 . No parameter adjustment was used to fit the data set, such that the theoretical predictions are independent estimates. In good agreement with the data, the theory suggests that the dependence $D=f(a d)$ is weak for low ad owing to the dominance of $D_{s}$ in this range. As $a d$ is increased, $D_{v}$ surpasses $D_{s}$, hence the sharp increase in $D$ observed for the high ad case.

numbers studied by Duan \& Wiggins, the experimental values given in table 1 were interpolated from available observations at $R e=90,140$ and 600 (shown in figure 8).

Included in table 1 is the contribution to dispersion predicted by the potential flow theory of Eames \& Bush (1999). For solid impermeable cylinders, the theory predicts a dispersive component, which we denote $D_{p}$, of magnitude

$$
D_{p}=0.74 \alpha U_{o} d .
$$

As shown in table $1, D_{p}$ is negligible compared to vortex trapping and secondarywake dispersion, indicating that for $R e \approx 10-1000$ the cylinder wakes are much more important to longitudinal dispersion than the effect of streamline curvature around cylinders described by the potential flow theory. Table 1 also shows that the dispersive component from the gap flow, (65), is likewise negligibly small compared with vortex trapping and secondary-wake dispersion.

In addition to results in table 1, a graphical comparison of theory and experiment for $R e=100$ and $R e=190$ is shown in figure 11. First, consider $R e=100$. The agreement is satisfactory and shows that the theory captures the weak addependence for low $a d$ and the sharp increase for high $a d$. As theory suggests, for low 
$a d$, secondary-wake dispersion makes the primary contribution to total dispersion $\left(D_{s}>D_{v}\right.$ in table 1 for $\left.R e=100, a d=0.013\right)$. However, vortex trapping becomes dominant as $a d$ is increased $\left(D_{v}>D_{s}\right.$ in table 1 for $\left.R e=100, a d=0.082\right)$. Note that for $R e=100$, we expect little or no contribution from turbulent mixing to total dispersion, in accordance with the results shown in figure 9, from which we estimated $D_{t} / U_{o} d<0.1$ for Reynolds numbers in the range 65-140, $a d=0.013$. For this $a d$, the sum $\left(D_{s}+D_{v}\right) / U_{o} d=1.38$, clearly much greater than the turbulent contribution.

For $R e=190$, the comparison between theory and experiment is also satisfactory, with the $a d$-dependence captured by the theory. However, the theory underpredicts the experimental observation at high $a d$. This may be attributed to the proximity of this Reynolds number to the turbulence transition. The increased mixing over small scales observed above $R e=140$ in figure 9 and the observation of a critical increase in turbulent mixing in the range near $R e=190$ by Nepf et al. (1997) would support a contribution from turbulent diffusion at this Reynolds number. Moreover, the estimate of the turbulent diffusivity based on figure 9 and given in the previous section is $O(0.1)$. This magnitude could account for the 0.35 underprediction shown in table 1 and figure $11(b)$ for $a d=0.082$.

\section{Conclusions}

We have presented a theoretical framework for predicting longitudinal dispersion in an array of randomly distributed cylinders at moderate Reynolds numbers characterized by unsteady cylinder wakes. We find that two processes predominantly contribute to the dispersion in this regime: trapping of tracer in the primary wakes and the advection of tracer through the spatially random velocity field created by cylinder secondary wakes. The vortex-trapping dispersion increases with the cylinder density, and is inversely dependent on Reynolds number, which dictates the features of the primary wake. Secondary-wake dispersion arises from the perturbation in mean velocity created by each cylinder. When superimposed, the randomly distributed perturbations create a randomly heterogeneous time-averaged velocity field. The variance of this velocity field is directly related to the array density. However, because the correlation length scale of the velocity field is inversely related to the array density, the effects cancel, and as such, secondary-wake dispersion is only weakly dependent on cylinder density. Qualitatively, this separates secondary-wake dispersion from vortextrapping dispersion, since the latter increases with density, while the former remains approximately constant.

The two types of dispersion described here should be intrinsic to flow through any array of distributed bodies at moderate to high Reynolds number, and extension of the theory for these two mechanisms to more complex morphology should be straightforward. That is, for sufficiently high Reynolds number and sufficiently far downstream of the body, the velocity defect becomes insensitive to the particular body morphology, and it is dictated primarily by the form drag. Thus, given the drag for a body, the associated velocity defect can be found, and used in (64) to predict dispersion due to the random velocity field. Further, one can predict the trapping dispersion given the residence time and size of the trapping region around any arbitrarily shaped body. That the framework presented in this paper can apply to general arrays of bodies is advantageous for describing dispersion in natural systems such as wetlands, since plant morphology may vary considerably.

The results we obtain are valid for relatively small ad (ミ0.1). For higher density, vortex-trapping dispersion is expected to be the dominant contributor to total 
dispersion, with trapping zones growing larger, and encompassing multiple cylinders. However, given the residence time parameters for these multi-body trapping zones, dispersion may be predicted by the theory presented here even for $a d>0.1$.

Our experimental observations indicate that a mixing transition occurs near $R e \approx$ 200. For smaller Reynolds numbers, the concentration field exhibits significant spatial heterogeneity, with distinct filaments of tracer created by the bending and stretching of tracer around the cylinders. Above $R e \approx 200$, the cylinder wakes contributes sufficient small-scale turbulence to erase the filament signatures and the tracer cloud appears smooth. Thus, while we have shown that turbulent diffusion does not contribute significantly to longitudinal dispersion, it is important to the local character of the tracer concentration field, which may be important to local mass and heat transfer processes.

Finally, it is pertinent to address the lack of experimental or numerical studies of Lagrangian transport in the oscillating primary wake behind circular cylinders. Nearly all research on primary wakes has been devoted to Eulerian mixing properties, e.g. turbulent fluctuations, and so the primary wake parameters such as the size and residence time, which are necessary to calculate vortex-trapping dispersion, are lacking. The dearth of literature on this subject is curious, given the general interest in Lagrangian mixing. With the exception of the work by MacLennan \& Vincent (1982), the only such studies, to our knowledge, of Lagrangian transport in cylinder wakes have come from dynamical systems applications (Ziemniak, Jung \& Tell 1994; Duan \& Wiggins 1997; Shariff, Pulliam \& Ottino 1991). In addition to being important to the prediction of dispersion in cylinder arrays, observations of Lagrangian transport in cylinder wakes is of fundamental interest, and we stress the need for more such studies.

\section{Appendix. Exact expression for dispersion due to the secondary wake velocity disturbance}

The dispersion constant due to $\left\langle\boldsymbol{u}^{\prime} \boldsymbol{c}^{\prime}\right\rangle$, the spatially heterogeneous velocity field, is determined as follows. Assuming, subject to confirmation, that the average flux due to spatial correlations behaves as a Fickian dispersive flux, i.e. is proportional to the mean concentration gradient, then we can write

$$
-D_{s} \nabla C_{o}=\left\langle u^{\prime} c^{\prime}\right\rangle \equiv \lim _{t \rightarrow \infty} \lim _{y \rightarrow \infty} \frac{1}{t} \int_{0}^{t} \mathrm{~d} t \int_{-\infty}^{x} P\left(\boldsymbol{x}_{1}\right) u^{\prime}\left(\boldsymbol{x} \mid \boldsymbol{x}_{1}, t\right) c^{\prime}\left(\boldsymbol{x} \mid \boldsymbol{x}_{1}, t\right) \mathrm{d} \boldsymbol{x}_{1} .
$$

The integral expression is simply the average over all spatial correlations, $u^{\prime} c^{\prime}(\boldsymbol{x}, t)$, due to fluctuations in the mean velocity and concentration fields caused by a cylinder held fixed at $\boldsymbol{x}=\boldsymbol{x}_{1}$. Here, the probability of finding a cylinder at $\boldsymbol{x}_{1}$ is the twodimensional probability density function $P\left(\boldsymbol{x}_{1}\right)=a / d$. The concentration fluctuation, $c^{\prime}\left(\boldsymbol{x} \mid \boldsymbol{x}_{1}, t\right)$ can be expressed as

$$
\begin{gathered}
c^{\prime}\left(\boldsymbol{x} \mid \boldsymbol{x}_{1}, t\right)=\int_{x_{1}+l_{r}}^{x(t)} \frac{U_{o} \nabla C_{o}-u\left(\boldsymbol{x}^{\prime}(t-\tau) \mid \boldsymbol{x}_{1}\right) \nabla C_{o} \mathrm{~d} x^{\prime}}{u\left(\boldsymbol{x}^{\prime}(t-\tau) \mid \boldsymbol{x}_{1}\right)}=\nabla C_{o} \int_{x_{1}+l_{r}}^{x(t)}-\frac{u^{\prime}\left(\boldsymbol{x}^{\prime}(t-\tau) \mid \boldsymbol{x}_{1}\right) \mathrm{d} x^{\prime}}{u\left(\boldsymbol{x}^{\prime}(t-\tau) \mid \boldsymbol{x}_{1}\right)}, \\
\mathrm{d} \boldsymbol{x}^{\prime}(t-\tau) / \mathrm{d} \tau=-\boldsymbol{u}\left(\boldsymbol{x}^{\prime} \mid \boldsymbol{x}_{1}, t-\tau\right), \quad \boldsymbol{x}^{\prime}(t)=\boldsymbol{x}(t),
\end{gathered}
$$

Specifically, $c^{\prime}(x, t)$ is due to the difference between the mean advective flux, $U_{o} \nabla C_{o}$, which is oriented in the streamwise direction, and the local advective flux, $u\left(\boldsymbol{x}^{\prime} \mid \boldsymbol{x}_{1}, t\right) \nabla C_{o}$ integrated over the path of a Lagrangian fluid parcel between $\boldsymbol{x}_{1}$ and 
$\boldsymbol{x}$. Note that while the velocity disturbance is a function of the two-dimensional coordinate, $\boldsymbol{x}^{\prime}, u$ is the streamwise component of the velocity disturbance, and the integration is over only the streamwise coordinate, $x$. Since we separate the flux due to the velocity disturbance in the secondary wake from the vortex trapping flux in the primary wake, integration is only performed between $x$ and $x_{1}+l_{r}$, the end of the recirculation zone (see figure 1). Moreover, the position variable, $\boldsymbol{x}^{\prime}(t-\tau)$, and thereby the local velocity, $\boldsymbol{u}\left(\boldsymbol{x}^{\prime} \mid \boldsymbol{x}_{1}, t-\tau\right)$, is enslaved to the time variable by the kinematic initial value problem (A 3 ). As a result, the time-dependent velocity disturbance, $\boldsymbol{u}\left(\boldsymbol{x} \mid \boldsymbol{x}_{1}, t\right)$, must be known to exactly calculate (A 2), and thus the problem is intractable unless approximations can be made, as in $\S 4$. Combining (A 1) and (A 2) gives

$$
D_{s}=\lim _{t \rightarrow \infty} \lim _{y \rightarrow \infty} \frac{1}{t} \frac{a}{d} \int_{0}^{t} \mathrm{~d} t \int_{-\infty}^{x} \mathrm{~d} \boldsymbol{x}_{1} u^{\prime}\left(\boldsymbol{x} \mid \boldsymbol{x}_{1}, t\right) \int_{x_{1}+l_{r}}^{x(t)} \frac{u^{\prime}\left(\boldsymbol{x}^{\prime}(t-\tau) \mid \boldsymbol{x}_{1}\right) \mathrm{d} x^{\prime}}{u\left(\boldsymbol{x}^{\prime}(t-\tau) \mid \boldsymbol{x}_{1}\right)},
$$

which must be solved with the aid of (A 3).

Provided the velocity disturbance, $u^{\prime}$, vanishes sufficiently fast for long distances away from the fixed cylinder at $x_{1}$, the integral in (A 4) will be independent of $x$, signifying that dispersion is non-local, and thus a finite constant value for $D_{s}$ results. As shown in $\S 4.1$, the decay of the velocity disturbance, $u^{\prime}$, is ensured by the resistance due to the remaining cylinders in the array, and there is an effective attenuation length, $\left(a C_{D}\right)^{-1}$, much like the Brinkman screening length in a viscous porous medium (Koch \& Brady 1985), which ensures the existence of $D_{s}$.

\section{REFERENCES}

Ayaz, F. \& Pedley, T. J. 1999 Flow through and particle interception by an infinite array of closely-spaced circular cylinders. Eur. J. Mech. B/Fluids 18, 173-196.

Bradshaw, P., Dean, R. B. \& McEligot, N. P. 1973 Calculation of interacting turbulent shear layers: duct flow. Trans. ASME I: J. Fluids Engng 95, 214-219.

Csanady, G. T. 1973 Turbulent Diffusion in the Environment. D. Reidel, Dordrecht, Holland.

Dagan, G. 1987 Theory of solute transport by groundwater. Annu. Rev. Fluid Mech. 19, 183-215.

DuAn, J. \& Wiggins, S. 1997 Lagrangian transport and chaos in the near wake of the flow around an obstacle: a numerical implementation of lobe dynamics. Nonlinear Processes Geophy. 4, $125-136$.

Eames, I. \& Bush, J. W. M. 1999 Longitudinal dispersion by bodies fixed in potential flow. Proc. R. Soc. Lond. A 455, 3665-3686.

Gerrard, J. H. 1978 The wakes of cylindrical bluff bodies at low Reynolds number. Phil. Trans. R. Soc. Lond. A 288, 351-382.

HiLl, R. J. \& KocH, D. L. 2002 Moderate-Reynolds-number flow in a wall-bounded porous medium. J. Fluid Mech. 453, 315-344.

HiNCH, E. J. 1977 An averaged-equation approach to particle interactions in a fluid suspension. J. Fluid Mech. 83, 695-720.

HinZE, J. O. 1975 Turbulence. McGraw-Hill.

Howells, I. D. 1974 Drag due to the motion of a Newtonian fluid through a sparse random array of small fixed rigid objects. J. Fluid Mech. 64, 449-475.

Hughes, B. D. 1995 Random Walks and Random Environments V1. Clarendon.

Koch, D. L. \& Brady, J. F. 1985 Dispersion in fixed beds. J. Fluid Mech. 154, 399-427.

Koch, D. L., Hill, R. J. \& SANGANi, A. S. 1998 Brinkman screening and the covariance of the fluid velocity in fixed beds. Phys. Fluids A 10, 3035-3037.

Koch, D. L. \& LADD, A. J. C. 1997 Moderate Reynolds number flows through periodic and random arrays of aligned cylinders. J. Fluid Mech. 349, 31-66.

KovasznaY, L. S. G. 1949 Hot-wire investigation of the wake behind cylinders at low Reynolds numbers. Proc. R. Soc. Lond. A 198, 174-190. 
Kundu, P. 1990 Fluid Mechanics. Academic.

LeVenspiel, O. \& SMith, W. K. 1956 Notes on the diffusion-type model for the longitudinal mixing of fluids in flow. Chem. Engng Sci. 6, 227-233.

MacLennan, A. S. M. \& Vincent, J. H. 1982 Transport in near aerodynamic wakes of flat plates. J. Fluid Mech. 120, 185-197.

NePf, H. M. 1999 Drag, turbulence, and diffusion in flow through emergent vegetation. Water Resour. Res. 35, 479-489.

Nepf, H. M., Sullivan, J. A. \& Zavistoski, R. A. 1997 A model for diffusion within emergent vegetation. Limnol. Oceanogr. 42, 1735-1745.

Paranthoen, P., Browne, L. W. B., Masson, S. Le, Dumouchel, F. \& Lecordier, J. C. 1999 Characteristics of the near wake of a cylinder at low Reynolds numbers. Eur. J. Mech. B/Fluids 18, 659-674.

Rehab, H., Antonia, R. A. \& Djenidi, J. Mi. 2000 Characteristics of fluorescein dye and temperature fluctuations in a turbulent near-wake. Exps. Fluids 28, 462-470.

Schlichting, H. 1987 Boundary-Layer Theory. McGraw-Hill.

Shariff, K., Pulliam, T. H. \& Ottino, J. M. 1991 A dynamical systems analysis of kinematics in the time-periodic wake of a circular cylinder. Lectures Appl. Math. 28, 613-646.

TAYLOR, J. R. 1997 Introduction to Error Analysis. University Science Books.

WeEks, E. R. \& Swinney, H. L. 1998 Anomalous diffusion resulting from strongly asymmetric random walks. Phys. Rev. E 57, 4915-4920.

Young, W. R. 1988 Arrested shear dispersion and other models of anomalous diffusion. J. Fluid Mech. 193, 129-149.

Young, W. R. \& Jones S. W. 1991 Dispersion in an unconsolidated porous medium. Phys. Fluids A 3, 2468-2470.

ZAVISTOSKI, R. A. 1994 Hydrodynamic effects of surface piercing plants. MS thesis, MIT, Cambridge, USA.

ZhANG, H. J. \& ZHOU, Y. 2001 Effect of unequal cylinder spacing on vortex streets behind three side-by-side cylinders. Phys. Fluids 13, 3675-3686.

Zhou, Y., So, R. M. C., Liu, M. H. \& Zhang, H. J. 2000 Complex turbulent wakes generated by two and three side-by-side cylinders. Intl J. Heat Fluid Flow 21, 125-133.

ZiemniaK, E. M., Jung, C., Tell, T. 1994 Tracer dynamics in open hydrodynamical flows as chaotic scattering. Physica D 176, 123-146. 